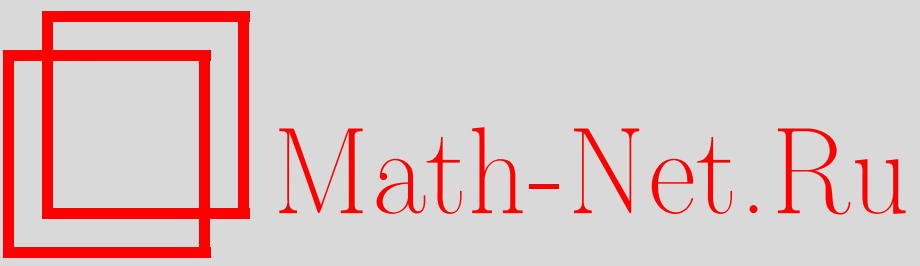

А. Г. Пинус, Условные термы и их приложения в алгебре и в теории вычислений, УМH, 2001, том 56, выпуск 4, 35-72

DOI: https://doi.org/10.4213/rm415

Использование Общероссийского математического портала Math-Net.Ru подразумевает, что вы прочитали и согласны с пользовательским соглашением

http://www.mathnet.ru/rus/agreement

Параметры загрузки:

IP: 54.198 .64 .247

26 апреля 2023 г., 14:57:03 


\title{
УСЛОВНЫЕ ТЕРМЫ И ИХ ПРИЛОЖЕНИЯ В АЛГЕБРЕ И В ТЕОРИИ ВЫЧИСЛЕНИЙ
}

\author{
А. Г. Пинус
}

На основе понятия условного терма формализуется понятие программно вьчислимой функции на универсальной алгебре. Это даёт некоторый новый подход к изучению традиционных алгебраических вопросов относительно как универсальных классов, так и отдельных конечных алгебр, а также возможность постановки и исследования вопроса о вычислительных возможностях универсальных алгебр.

Библиография: 66 названий.

\section{СОДЕРЖАНИЕ}

Глава 1. Рационально и Морита-эквивалентные многообразия алгебр.

Дискриминаторные многообразия

1. Рационалшная эквивалентность многообразий алгебр ............... 37

2. Морита-эквивалентные многообразия алгебр .................... 38

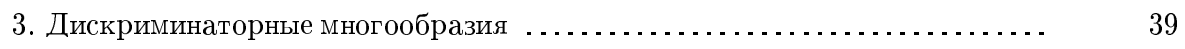

4. Представление инверсных полугрупп .............................. 43

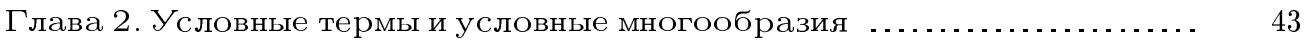

1. Условные термы и условно термальные функции ................... 43

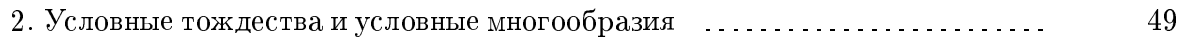

3. Исчисление условных тождеств ............................... 50

Глава 3. Условно рациональная эквивалентность и связанные с ней во-

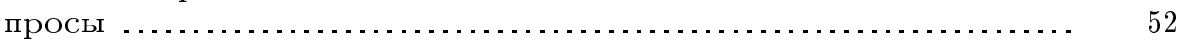

1. Условно рациональная эквивалентность и категории вложимости $\ldots . . . .52$

2. Сколемовские и условно термальные функции ....................

3. Морита-эквивалентность по вложимости .......................... 55

4. Определимость алгебр производньми структурами ................ 55

Глава 4. Инварианты отношений условной рациональной эквивалентности и схожести ........................................... 57

1. Инварианты отношения условной рациональной эквивалентности конечных

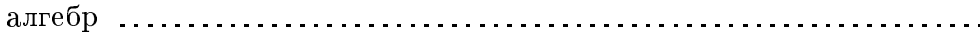

2. Инварианты отношения условной рационалњной эквивалентности йонссоновских локально конечных многообразий ...................... 58

3. Инварианты отношения схожести между конечными алгебрами . . . . . . . 6

Глава 5. Классификация конечных алгебр по их вычислительным возможностям 


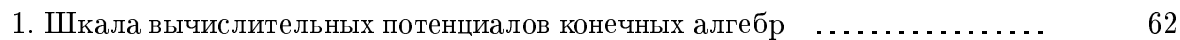

2. Алгебры, условно рационально эквивалентные классическим алгебрам . 63

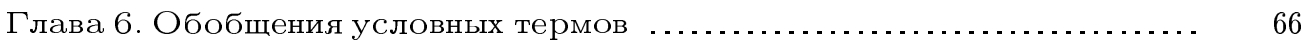

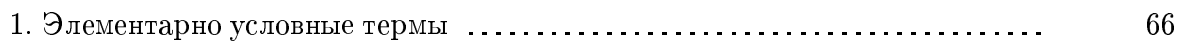

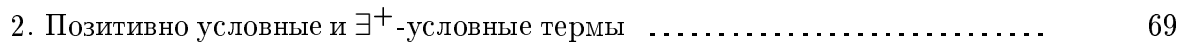

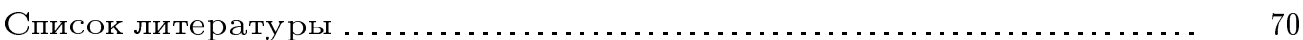

Одной из возможных прикладных трактовок понятий универсальной алгебры и терма сигнатуры этой алгебры является следующая: на некоторой совокупности объектов, основном множестве универсальной алгебры, заданы некоторые стандартные (будем далее назьвать их простейшими) программы преобразований, вычислений, соответствующие сигнатурным функциям данной универсальной алгебры. Тогда понятие терма сигнатуры этой алгебры можно рассматривать как некоторую программу преобразований, выгислений на основном множестве, составленную из простейших (сигнатурных) программ с помощью лишь оператора суперпозиции. Однако в программировании используется еще цельй ряд принципов композиции более сложных программ из подпрограмм, в том числе и так назьваемьй условный оператор. Автором данного обзора было предпринято рассмотрение абстрактного понятия условного оператора в рамках теории универсальных алгебр и на основе этого понятия было предложено понятие условного терма. Интуитивно концепщия условного терма соответствует понятию программы вычислений, преобразований на основном множестве универсальной алгебры, составленной из простейших (сигнатурных) программ с помощью оператора суперпозиции и условного оператора. Изучение понятия условного терма оказалось достаточно плодотворным для решения целого ряда естественных внутренних вопросов универсальной алгебры: описание программно вычислимых функций на алгебре, описание функций, коммутирующих с полугруппами преобразований алгебр, описание сколемовских функций, различные вопросы классификации конечных и локально конечных универсальных алгебр, изучение категорий вложимости универсальных классов универсальных алгебр, производных структур как инвариантов той или иной классификации алгебр и т. д. С другой стороны, полученные результаты находят некоторую естественную интерпретацию в теории программ вычислений, о чем уже говорилось выше. Понятие условного терма позволило найти некоторьй подход к активному изучению строения универсальных классов универсальных алгебр, а значит, и отдельных конечных алгебр, который невозможен в рамках традиционных методов изучения многообразий алгебр.

Настоящий обзор посвящен изложению основных результатов, связанных с понятием условного терма. Используются стандартные обозначения и понятия универсальной алгебры, которые можно найти в монограбриях [9], [13], [15], [21], [23], [25], [32], [33], [62], [8], [17], [28], [52], [61] и обзоре [1]. Далее, для простоты, будем рассматривать алгебры не более чем счетной сигнатуры. Важную роль в доказательствах утверждений об условных термах играют результаты А.И. Мальцева о рационально эквивалентных многообразиях, Р. Мак-Кензи о Морита-эквивалентных многообразиях и теория дискриминаторных многообразий, отсутствующие в указанной литературе. В связи с этим настояший обзор начинается с изложения этих результатов.

Работа выполнена при финансовой поддержке Российского фонда фундаментальных исследований (грант № 99-01-00571). 


\section{Глава 1.}

Рационально и Морита-эквивалентные многообразия алгебр. Дискриминаторные многообразия

1. Рациональная эквивалентность многообразий алгебр. Прежде всего, напомним, что многообразием универсальных алгебр называется произвольньй класс $\mathscr{M}$-универсальных алгебр фиксированной сигнатуры $\sigma$, замкнутьй относительно перехода к подалгебрам, гомоморфньм образам, и образования прямых произведений. В силу известной теоремы Г. Биркгофа многообразия алгебр суть эквациональные классы алгебр, т.е. классы всех алгебр данной сигнатуры, на которых истинна некоторая система тожсдеств.

Далее, говоря о классах универсальных алгебр, будем иметь в виду классы алгебр некоторой фиксированной сигнатуры.

ОПРЕДЕлЕниЕ 1.1. а) Для любого класса $\mathscr{K}$ универсальных алгебр через $\overrightarrow{\mathscr{K}}$ обозначим категорию, объектами которой являются $\mathscr{K}$-алгебры, а морфизмами - гомоморфизмы между $\mathscr{K}$-алгебрами.

б) Две категории $\overrightarrow{\mathscr{K}}_{1}$ и $\overrightarrow{\mathscr{K}}_{2}$ назовем изоморфнылми, если они изоморфны как частичные полугруппы или, что равносильно, если существуют биекция $\varphi$ совокупности объектов $\mathrm{Ob}\left(\overrightarrow{\mathscr{K}}_{1}\right)$ категории $\overrightarrow{\mathscr{K}}_{1}$ на совокупность объектов $\mathrm{Ob}\left(\overrightarrow{\mathscr{K}}_{2}\right)$ категории $\overrightarrow{\mathscr{K}}_{2}$ и, для любых $a, b \in \mathrm{Ob}\left(\overrightarrow{\mathscr{K}}_{1}\right)$, биекция $\varphi_{a, b}$ множества морфизмов $\operatorname{Hom}_{\overrightarrow{\mathscr{K}}_{1}}(a, b)$ из $a$ в $b$ на множество морфизмов $\operatorname{Hom}_{\overrightarrow{\mathscr{K}}_{2}}(\varphi(a), \varphi(b))$ такие, что для любых $a, b, c \in \mathrm{Ob}\left(\overrightarrow{\mathscr{K}}_{1}\right)$ и любых $\alpha \in \operatorname{Hom}_{\mathscr{K}_{1}}(a, b), \beta \in \operatorname{Hom}_{\mathscr{K}_{1}}(b, c)$ имеет место равенство $\varphi_{a, c}(\beta \alpha)=\varphi_{a, b}(\beta) \varphi_{b, c}(\alpha)$. Изоморфизм категорий $\overrightarrow{\mathscr{K}}_{1}$ и $\overrightarrow{\mathscr{K}}_{2}$ обозначим как $\overrightarrow{\mathscr{K}}_{1} \cong \overrightarrow{\mathscr{K}}_{2}$.

в) Два класса алгебр $\mathscr{K}_{1}$ и $\mathscr{K}_{2}$ назовем Морита-әквивалентнылми, если изоморфны категории $\overrightarrow{\mathscr{K}}_{1}$ и $\overrightarrow{\mathscr{K}}_{2}$.

г) Через $\overrightarrow{\text { Set } о б о з н а ч и м ~ к а т е г о р и ю ~ м н о ж е с т в ~(о б ъ е к т ы ~} \overrightarrow{\mathrm{Set}}-$ любые множества, морфизмы - любые отображения одного множества в другое). Под стирающим функтором $S_{\overparen{K}}$ для любого класса универсальных алгебр $\mathscr{K}$ будем понимать функтор из категории $\overrightarrow{\mathscr{K}}$ в категорию $\overrightarrow{\mathrm{Set}}$, сопоставляющий универсальной алгебре ее основное множество, а гомоморфизму между $\mathscr{K}$-алгебрами - соответствуюшее отображение основных множеств этих алгебр.

д) Классы $\mathscr{K}_{1}$ и $\mathscr{K}_{2}$ универсальных алгебр назовем әквивалентнылми, если существует изоморфизм $\varphi$ категории $\overrightarrow{\mathscr{K}}_{1}$ на категорию $\overrightarrow{\mathscr{K}}_{2}$, коммутируюший со стираюшими функторами категорий $\overrightarrow{\mathscr{K}}_{1}$ и $\overrightarrow{\mathscr{K}}_{2}: S_{\overrightarrow{\mathscr{K}}_{1}} \varphi=S_{\mathscr{K}_{2}}$. Эквивалентность классов $\mathscr{K}_{1}$ и $\mathscr{K}_{2}$ обозначим как $\mathscr{K}_{1} \simeq \mathscr{K}_{2}$.

ОПРЕДЕЛЕНИЕ 1.2. Классы $\mathscr{K}_{1}$ алгебр сигнатуры $\sigma_{1}$ и $\mathscr{K}_{2}$ сигнатуры $\sigma_{2}$ назовем рационально эквивалентными, если существуют отображения $F_{1}\left(F_{2}\right)$, сопоставляюшие символам сигнатуры $\sigma_{1}$ термы сигнатуры $\sigma_{2}$ (символам сигнатуры $\sigma_{2}$ термы сигнатуры $\left.\sigma_{1}\right)$ с сохранением арности (числа аргументов функции $f \in \sigma_{i}$ и терма $F_{i}(f)$ совпадают) и такие, что:

а) для любой $\mathscr{K}_{1}$-алгебры $\mathscr{A}=\left\langle A ; \sigma_{1}\right\rangle$ алгебра $F_{2}(\mathscr{A})=\left\langle A ; \sigma_{2}\right\rangle$ входит в класс 
$\mathscr{K}_{2}$, здесь $\sigma_{2}$-операшии алгебры $F_{2}(\mathscr{A})$ определяются на $A$ соответствуюшими $F_{2}\left(\sigma_{2}\right)$-термами алгебры $\mathscr{A}$;

б) для любой $\mathscr{K}_{2}$-алгебры $\mathscr{A}=\left\langle A ; \sigma_{2}\right\rangle$ алгебра $F_{1}(\mathscr{A})=\left\langle A ; \sigma_{1}\right\rangle$ входит в класс $\mathscr{K}_{1}$;

в) для любой $\mathscr{K}_{1}$-алгебры $\mathscr{A}$ имеет место равенство $F_{1}\left(F_{2}(\mathscr{A})\right)=\mathscr{A}$;

г) для любой $\mathscr{K}_{2}$-алгебры $\mathscr{A}$ имеет место равенство $F_{2}\left(F_{1}(\mathscr{A})\right)=\mathscr{A}$.

Рациональную эквивалентность классов $\mathscr{K}_{1}$ и $\mathscr{K}_{2}$ обозначим как $\mathscr{K}_{1} \sim \mathscr{K}_{2}$. Говоря о рациональной эквивалентности алгебр $\mathscr{A}_{1}$ и $\mathscr{A}_{2}$, будем иметь в виду рациональную эквивалентность классов $I\left(\left\{\mathscr{A}_{1}\right\}\right)$ и $I\left(\left\{\mathscr{A}_{2}\right\}\right)$. Здесь и далее $I(\mathscr{K})=\{\mathscr{B} \mid \mathscr{B} \cong \mathscr{A}$ для некоторой $\mathscr{A} \in \mathscr{K}\}$. Пусть $\mathscr{F} \mathscr{M}(X)$ - это $\mathscr{M}$-свободная $X$-порожденная $\mathscr{M}$-алгебра для любого многообразия $\mathscr{M}$ универсальных алгебр.

Имеет место следуюшая связь между введенными понятиями, установленная А.И. Мальцевым.

Теорема 1.1 [24]. Для любьх многообразий алгебр $\mathscr{M}_{1}$ и $\mathscr{M}_{2}$ следующие условия эквивалентны:

a) $\mathscr{M}_{1} \simeq \mathscr{M}_{2}$;

б) $\mathscr{F} \mathscr{M}_{1}\left(\aleph_{0}\right) \sim \mathscr{F} \mathscr{M}_{2}\left(\aleph_{0}\right)$;

в) существуют алгебрь $\mathscr{A}_{1} \in \mathscr{M}_{1}$ и $\mathscr{A}_{2} \in \mathscr{M}_{2}$ такие, что $\mathscr{M}_{1}=\mathscr{M}\left(\mathscr{A}_{1}\right)$, $\mathscr{M}_{2}=\mathscr{M}\left(\mathscr{A}_{2}\right)$ u $\mathscr{A}_{1} \sim \mathscr{A}_{2}$

г) $\mathscr{M}_{1} \sim \mathscr{M}_{2}$.

Здесь и далее $\mathscr{M}(\mathscr{K})$ - это многообразие, порожденное классом $\mathscr{K}$ универсальных алгебр (наименьшее многообразие, включающее в себя класс $\mathscr{K}$ ).

2. Морита-эквивалентные многообразия алгебр. Из определений 1.1 и 1.2 очевидно, что рациональная эквивалентность многообразий $\mathscr{M}_{1}$ и $\mathscr{M}_{2}$ влечет их Морита-эквивалентность. Обратное неверно. Это, в частности, вытекает из следующей известной теоремы Т. К. Ху.

Tеорема 1.2 [18]. Многообразие $\mathscr{M}$ универсальных алгебр Морита-эквивалентно многообразию $B$ булевых алгебр тогда и только тогда, когда многообразие $\mathscr{M}$ порождено некоторой примальной алгеброй.

В связи с этим напомним, что алгебра $\mathscr{A}$ назьвается примальной, если она конечна и любая функция на ее основном множестве является термальной.

ОПРЕДЕЛЕНИЕ 1.3. Пусть $\mathscr{A}$ - некоторая универсальная алгебра и $\eta(x)$ - одноместньй терм сигнатуры алгебры $\mathscr{A}$. Терм $\eta(x)$ называется идемпотентныл на $\mathscr{A}$, если на $\mathscr{A}$ истинно тождество $\eta(\eta(x))=\eta(x)$. Через $\mathscr{A}(\eta)$ обозначим алгебру, основным множеством которой является множество $\eta(\mathscr{A})=\{a \in \mathscr{A} \mid \eta(a)=a\}$ и сигнатура которой состоит из функций вида $t_{\eta}$, где $t\left(x_{1}, \ldots, x_{m}\right)$ - произвольньй $m$-местный терм сигнатуры алгебры $\mathscr{A}$, при этом функция $t_{\eta}$ также $m$-местна и для $\bar{a} \in \eta(\mathscr{A})$ $t_{\eta}(\bar{a})=\eta(t(\bar{a}))$. Алгебру $\mathscr{A}(\eta)$ назовем $\eta$-редуктом алгебры $\mathscr{A}$. Если $\mathscr{K}-$ некоторьй класс универсальных алгебр и $\mathscr{K} \models \eta(\eta(x))=\eta(x)$, то пусть $\mathscr{K}(\eta)=\{\mathscr{B} \mid \mathscr{B} \cong \mathscr{A}(\eta)$ для некоторой $\mathscr{A} \in \mathscr{K}\}$. Если $\sigma-$ сигнатура алгебры $\mathscr{A}$, то через $\sigma_{\eta}$ обозначим сигнатуру алгебры $\mathscr{A}(\eta)$.

Нетрудно заметить, что если $\eta(x)$ - идемпотентный терм на многообразии $\mathscr{M}$, то и класс $\mathscr{M}(\eta)$ является многообразием. 
ОПРЕДЕЛЕНИЕ 1.4. Пусть $\mathscr{K}$ - некоторьй класс универсальных алгебр и $\eta(x)-$ одноместный терм сигнатуры класса $\mathscr{K}$. Терм $\eta(x)$ назьвается обратимылм на $\mathscr{K}$, если для некоторого натурального $n$ сушествуют одноместные термы $t_{1}(x), \ldots, t_{n}(x)$ и $n$-местный терм $t\left(x_{1}, \ldots, x_{n}\right)$ сигнатуры класса $\mathscr{K}$ такие, что

$$
\mathscr{K} \models x=t\left(\eta\left(t_{1}(x)\right), \ldots, \eta\left(t_{n}(x)\right)\right) .
$$

ОПРЕДЕЛЕНИЕ 1.5. Для любой алгебры $\mathscr{A}=\langle A ; \sigma\rangle$ и любого $n \in \omega \backslash\{0\}$ под матричной степенью $\mathscr{A}^{[n]}$ алгебры $\mathscr{A}$ имеется в виду алгебра с основньм множеством $A^{n}$ и сигнатуры $\sigma^{[n]}$, состояшей из функциональных символов $m_{t}$ арности $r$, где $t=\left\langle t_{1}(\bar{x}), \ldots, t_{n}(\bar{x})\right\rangle$ - последовательность любых термов сигнатуры $\sigma$ от $r n$ переменных. При этом $r$-арная операция $m_{t}:\left(A^{n}\right)^{r}=A^{n r} \rightarrow A^{n}$ определена так, что для любого $i \leqslant r$ и любых $\bar{a} \in\left(A^{n}\right)^{r}=A^{n r}$ имеет место равенство $\pi_{i} m_{t}(\bar{a})=t_{i}(\bar{a})$. Здесь $\pi_{i}$-проекция $A^{n}$ на $i$-ю координату. Для произвольного класса $\mathscr{K}$ универсальных алгебр пусть $\mathscr{K}^{[n]}=I\left(\left\{\mathscr{A}^{[n]} \mid \mathscr{A} \in \mathscr{K}\right\}\right)$.

Довольно очевидно, что если $\mathscr{M}$ многообразие, то и $\mathscr{M}^{[n]}$ является многообразием для любого $n \in \omega \backslash\{0\}$. Без особого труда доказьваются также следуюшие утверждения:

для любого многообразия $\mathscr{M}$ и любого $n \in \omega \backslash\{0\}$ существует стандартный изоморфизм $F_{\mathscr{M}, n}$ категории $\overrightarrow{\mathscr{M}}$ на категорию $\overrightarrow{\mathscr{M}^{[n]}}$;

для любого многообразия $\mathscr{M}$ и любого обратимого идемпотентного терма $\eta(x)$ на $\mathscr{M}$ сушествует стандартный изоморфизм $F_{\mathscr{M}, \eta}$ категории $\overrightarrow{\mathscr{M}}$ на категорию $\overrightarrow{\mathscr{M}}(\eta)$.

Напомним также, что, как уже отмечалось выше, рационально эквивалентные многообразия имеют изоморфные категории. Следующий результат Р. Мак-Кензи показывает, что любая Морита-эквивалентность многообразий сводится к комбинации трех этих конструкций.

ТеОрема 1.3 [27]. Для любых многообразий $\mathscr{M}$ и $\mathcal{N}$ универсальньх алгебр если $F$ - изоморфизм категории $\overrightarrow{\mathscr{M}}$ на категорию $\overrightarrow{\mathcal{N}}$, то существуют $n \in \omega \backslash\{0\}$ и $\eta(x)$ - обратимый идемпотентный терм сигнатуры многообразия $\mathscr{M}^{[n]}$, такие, что изоморфизм F разлагается в композицию стандартных изоморфизмов $F_{\mathscr{M}, n}: \overrightarrow{\mathscr{M}} \rightarrow \overrightarrow{\mathscr{M}}^{[n]}, F_{\mathscr{M}^{[n], \eta}}: \overrightarrow{\mathscr{M}}^{[n]} \rightarrow \mathscr{M}^{[n]}(\eta)$ и некоторой рациональной әквивалентности многообразий $\mathscr{M}^{[n]}(\eta)$ u $\mathscr{N}$.

Некоторые частные вопросы, связанные с изоморфизмом категорий многообразий, рассмотрены в [2], [3].

3. Дискриминаторные многообразия. Основы теории дискриминаторных многообразий были заложены в работах А. Пиксли, Р. Квакенбуша, Х. Вернера, Р. Мак-Кензи, С. Барриса (см. [22], [60], [65]).

ОПРЕДЕЛЕНИЕ 1.6. Дискриминатором (тернарным) на множестве $A$ назьвается трехместная функция $d(x, y, z)$, определенная на $A$ и такая, что для любых $a, b, c \in A$

$$
d(a, b, c)= \begin{cases}a, & \text { если } a \neq b, \\ c, & \text { если } a=b .\end{cases}
$$


Нормальной трансформацией на множестве $A$ называется четырехместная функция $n(x, y, z, u)$ на $A$ такая, что для любых $a, b, c, e \in A$

$$
n(a, b, c, e)= \begin{cases}c, & \text { если } a=b, \\ e, & \text { если } a \neq b .\end{cases}
$$

Дискриминатор и нормальная трансформация, заданные на одном и том же множестве $A$, очевидным образом термально выразимы друг через друга:

$$
n(x, y, z, u)=d(d(x, y, z), d(x, y, u), u), \quad d(x, y, z)=n(x, y, z, x) .
$$

ОПРеДЕЛЕНИЕ 1.7. Универсальная алгебра $\mathscr{A}$ называется дискриминаторной алгеброй, если дискриминаторная функщия на основном множестве алгебры $\mathscr{A}$ является термальной функцией алгебры $\mathscr{A}$. Класс алгебр $\mathscr{K}$ называется дискриминаторнылм классом, если некоторьй терм сигнатуры класса $\mathscr{K}$ определяет дискриминаторные функции на основных множествах всех алгебр из $\mathscr{K}$. Многообразие универсальных алгебр назьвается дискриминаторным многообразием, если оно порождено некоторьм дискриминаторным классом алгебр.

Очевидно, что в силу определения любая примальная алгебра является дискриминаторной. В качестве примера приведем в явном виде определение дискриминатора на двухэлементной булевой алгебре $2=\langle\{0,1\} ; \wedge, \vee, \neg\rangle: d(x, y, z)=(x \wedge \neg(x \oplus y)) \vee(z \wedge$ $(x \oplus y))$, где $x \oplus y=(x \wedge \neg y) \vee(y \wedge \neg x)$. В силу известного результата Михлера и Вилли [29] многообразие колец является дискриминаторньм тогда и только тогда, когда оно порождено конечньм множеством конечных полей или, что то же самое, когда оно удовлетворяет тождеству $x^{n}=x$ для некоторого $n \in \omega$. В частности, если $\langle F ;+, \cdot\rangle$ - поле, состояшее из $n$ элементов, то терм

$$
t(x, y, z)=z+(x-z)(y-z)^{n-1}
$$

определяет дискриминатор на $F$. Этот ряд примеров дискриминаторных алгебр можно продолжить и далее.

Напомним, что алгебра $\mathscr{A}$ назьвается простой, если решетка Con $\mathscr{A}$ ее конгруэнций двухэлементна. Алгебра $\mathscr{A}$ наследственно проста, если проста любая ее неодноэлементная подалгебра. Очевидным образом замечается, что любая неодноэлементная дискриминаторная алгебра наследственно проста. Многообразие $\mathscr{M}$ конгруэни-перестановочно, если для любой алгебры $\mathscr{A}$ из $\mathscr{M}$ и любых конгруэнций $\Theta_{1}, \Theta_{2}$ на $\mathscr{A}$ имеет место равенство $\Theta_{1} \circ \Theta_{2}=\Theta_{2} \circ \Theta_{1}$. Многообразие $\mathscr{M}$ конгруэни-дистрибутивно, если для любой алгебры $\mathscr{A}$ из $\mathscr{M}$ решетка Con $\mathscr{A}$ дистрибутивна (их описания см. в [20]). В случае, когда $\mathscr{M}$ одновременно конгруэнц-перестановочно и конгруэнщ-дистрибутивно, говорят об арифметичности $\mathscr{M}$. Известно следуюшее описание арифметических многообразий с помощью мальцевских условий, принадлежашее А. Пиксли.

Tеорема 1.4 [56]. Многообразие $\mathscr{M}$ арифметично тогда и только тогда, когда существует терм $p(x, y, z)$ такой, что на $\mathscr{M}$ истиннь тождества

$$
p(x, y, x)=p(x, y, y)=p(y, y, x)=x .
$$


Пусть $\mathscr{M}$ - дискриминаторное многообразие, $\mathscr{M}=\mathscr{M}(\mathscr{K})$ и дискриминаторы на $\mathscr{K}$-алгебрах определены термом $t(x, y, z)$. Через $\mathscr{M}_{t}$ обозначим класс всех $\mathscr{M}$-алгебр, на которых $t(x, y, z)$ задает дискриминатор (в частности, $\mathscr{K} \subseteq \mathscr{M}_{t}$ ). Через $\mathscr{M}_{S I}$ для любого многообразия $\mathscr{M}$ обозначим класс всех подпрямо неразложимых $\mathscr{M}$-алгебр, а через $\mathscr{M}_{S}$ - класс всех простых $\mathscr{M}$-алгебр. Многообразие $\mathscr{M}$ назьвается полупросmылм, если $\mathscr{M}_{S I}=\mathscr{M}_{S}$. Алгебра $\mathscr{A}$ конгруэни-регулярна, если для любых $\Theta_{1}, \Theta_{2} \in$ Con $\mathscr{A}$ и любого $a \in \mathscr{A}$ из равенства $[a]_{\Theta_{1}}=[a]_{\Theta_{2}}$ вытекает равенство $\Theta_{1}=\Theta_{2}$. Многообразие $\mathscr{M}$, состояшее из конгруэнц-регулярных алгебр, назьвается конгруэнц-регулярньм. Алгебра $\mathscr{A}$ конгруэни-униформна, если для любой $\Theta \in$ Con $\mathscr{A}$ и любых $a, b \in \mathscr{A}$ мошности классов $[a]_{\Theta}$ и $[b]_{\Theta}$ совпадают. Многообразие $\mathscr{M}$ конгруэнц-униформно, если конгруэнш-униформны все $\mathscr{M}$-алгебры. Многообразие $\mathscr{M}$ обладает свойством продолжимости конгруәнций, если для любой $\mathscr{M}$-алгебры $\mathscr{A}$, любой ее подалгебры $\mathscr{A}^{\prime}$ и любой конгруэнции $\Theta^{\prime}$ на алгебре $\mathscr{A}^{\prime}$ существует некоторая конгруэнция $\Theta$ на $\mathscr{A}$, ограничение которой до алгебры $\mathscr{A}^{\prime}$ совпадает с $\Theta^{\prime}$. Многообразие $\mathscr{M}$ имеет эквационально определимые главные конгруэнции, если сушествует совокупность термов $t_{1}^{1}(x, y, u, v), t_{1}^{2}(x, y, u, v), \ldots, t_{n}^{1}(x, y, u, v), t_{n}^{2}(x, y, u, v)$ такая, что для любой алгебры $\mathscr{A}$, любых $a, b, c, d \in \mathscr{A}$ имеет место $\langle a, b\rangle \in \Theta_{c, d}^{\mathscr{A}} \leftrightarrow$ $\&_{i=1}^{n} t_{i}^{1}(a, b, c, d)=t_{i}^{2}(a, b, c, d)$. Здесь $\Theta_{c, d}^{\mathscr{A}}-$ главная конгруэнция на $\mathscr{A}$, порожденная парой $\langle c, d\rangle$.

Основные свойства дискриминаторных многообразий суммированы в виде следуюшего утверждения (различные части которого были доказаны в работах А. Пиксли, Р. Квакенбуша, Х. Вернера, С. Коммера, Р. Мак-Кензи, С. Барриса).

Теорема 1.5. Пусть $\mathscr{M}$ - дискриминаторное многообразие, тогда:

1) $\mathscr{M}$ арифметично, обладает свойством продолэимости конгруэниий, конгруэни-регулярно, конгруэнц-униформно, полупросто и имеет эквационально определимье конгруэнции (если терм $t(x, y, z)$ определяет дискриминаторы на некотором классе алгебр, порождающем $\mathscr{M}$, то для любой $\mathscr{A} \in \mathscr{M}$, любих $a, b, c, d \in \mathscr{A}$ включение $\langle c, d\rangle \in \Theta_{a, b}^{\mathscr{A}}$ равносильно равенству $\left.t(a, b, c)=t(a, b, d)\right)$;

2) если $\mathscr{M}=\mathscr{M}(\mathscr{K})$ и терм $t(x, y, z)$ определяет дискриминатор на $\mathscr{K}$-алгебрах, то $\mathscr{M}_{S}=\mathscr{M}_{S I}=\mathscr{M}_{t}$;

3) для любой $\mathscr{A} \in \mathscr{M}$ главнье конгруэнции алгебры $\mathscr{A}$ образуют подрешетку решетки Con $\mathcal{A}$, причем эта подрешетка - дистрибутивная решетка с относительными дополнениями.

Среди отдельных дискриминаторных алгебр наиболее изученными являются конечные алгебры.

ОПРЕДЕЛЕНИЕ 1.8. Конечные дискриминаторные алгебры называются квазипримальньими.

А. Пиксли были получены следующие описания квазипримальных алгебр.

Теорема 1.6 [57]. 1) Конечная алгебра А квазипримальна тогда и только тогда, когда многообразие $\mathscr{M}(\mathscr{A})$ арифметично и $\mathscr{A}$ наследственно проста.

2) Конечная алгебра $\mathscr{A}$ квазипримальна тогда и только тогда, когда термальной на $\mathscr{A}$ является любая функиия $f$, определенная на основном множестве алгебры $\mathcal{A}$, такая, что любая подалгебра алгебры $\mathscr{A}^{2}$, являюшаяся графиком изоморфизма между какими-либо подалгебрами алгебры $\mathscr{A}$, замкнута относительно $f$. 
О роли квазипримальных алгебр среди прочих можно судить, исходя из оценки их доли среди всех конечных алгебр. Пусть $G(n)$ - число всех попарно неизоморфных группидов мощности $n, Q(n)$ - число попарно неизоморфных квазипримальных группоидов мощности $n$. В. Л. Мурским была получена следующая оценка удельного веса квазипримальных группоидов среди всех.

Tеорема 1.7 [31]. $\lim _{n \rightarrow \infty} Q(n) / G(n)=1$, m.e. "почти все" конечные группоиды большой мощности квазипримальнь.

Каждая примальная алгебра очевидньм образом квазипримальна. А. Фостер и А. Пиксли выделили родовые признаки примальности для квазипримальных алгебр.

Теорема 1.8 [16]. Конечная алгебра $\mathscr{A}$ примальна тогда и только тогда, когда А⿱ квазипримальна, не имеет собственньх подалгебр и нетривиальных автоморфизмов.

Важную роль в работе с условными термами будет играть в дальнейшем и следующее описание дискриминаторных многообразий “почти мальцевскими условиями”, полученное Р. Мак-Кензи.

Теорема 1.9 [26]. Многообразие $\mathscr{M}$ дискриминаторно тогда и только тогда, когда для некоторого тернарного терма $t(x, y, z)$ на $\mathscr{M}$ истинны следующие тождества:

1) $t(x, x, y)=y, t(x, y, x)=x, t(x, y, y)=x$

2) $t(x, t(x, y, z), y)=y$;

3) $t\left(x, y, f\left(z_{1}, \ldots, z_{n}\right)\right)=t\left(x, y, f\left(t\left(x, y, z_{1}\right), \ldots, t\left(x, y, z_{n}\right)\right)\right)$, где $f-$ произвольный $п$-местный символ сигнатуры многообразия $\mathscr{M}$.

При этом если подобньй терм $t$ существует, то он является дискримина-

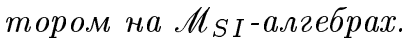

Большие возможности при работе с дискриминаторными многообразиями открывает представление алгебр этих многообразий в виде булевых конструкций из простых алгебр. Не останавливаясь на определениях булевых степеней, булевых произведений и прочего, которые можно найти, в частности, в специально посвященных им монографии [29] и обзоре автора [34], сформулируем лишь основные результаты, связанные с булевыми представлениями алгебр дискриминаторных многообразий. Через

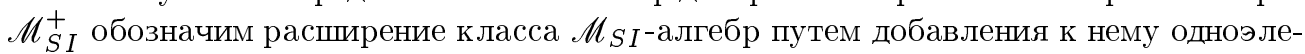
ментных алгебр. В работах С. Бульмана-Флеминга, Х. Вернера и Д. Кларка, П. Краусса получена следующая, в терминах булевых конструкций, характеризация дискриминаторных многообразий в классе всех конгруэнц-дистрибутивных многообразий.

ТЕОРема 1.10 [7], [11]. Для любого конгруэни-дистрибутивного многообразия М следующие условия әквивалентны:

1) $\mathscr{M}$ дискриминаторно,

2) любая $\mathscr{M}$-алгебра представима как булево произведение $\mathscr{M}_{S I}^{+}$-алгебр.

Описание всех (без условия конгруэнц-дистрибутивности) конечно порожденных (т.е. порожденных конечным числом конечных алгебр) многообразий $\mathscr{M}$, алгебры ко-

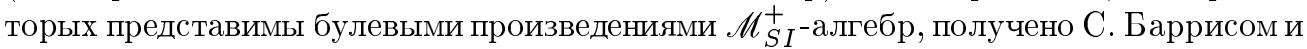
Р. Мак-Кензи. 
ТеОРема 1.11 [8]. Для любого многообразия $\mathscr{M}$ следующие условия эквивалентни:

1) для некоторой конечной совокупности $\mathscr{K}$ конечных $\mathscr{M}$-алгебр имеет место представление любой $\mathscr{M}$-алгебрь в виде булева произведения $\mathscr{K}$-алгебр;

2) $\mathscr{M}$ является прямым произведением многообразий $\mathscr{M}_{1}$ и $\mathscr{M}_{2}$ для некоторого конечно порожденного дискриминаторного многообразия $\mathscr{M}_{1}$ и некоторого конечно порохсденного абелева многообразия $\mathscr{M}_{2}$ такого, что кольцо $R\left(\mathscr{M}_{2}\right)$ имеет конечный тип представлений.

Представление алгебр дискриминаторного многообразия $\mathscr{M}$ в виде булева произведения $\mathscr{M}_{S I}^{+}$-алгебр позволяет достаточно конструктивно описывать строение совокупности всех гомоморфизмов из одной $\mathscr{M}$-алгебры в другую, что играет сушественную роль в работе с условными термами, в частности, в доказательстве теоремы 3.1.

Заметим также, что доказательства приведенных здесь теорем о булевых представлениях многообразий $\mathscr{M}$ построены на тщательном изучении строения решеток конгруэнций $\mathscr{M}$-алгебр.

В заключение этой главы остановимся еще на одном чисто полугрупповом результате, который будет востребован нами в дальнейшем.

4. Представление инверсных полугрупп. Напомним, что элементы $a$ и $b$ полугрупшы $\mathscr{S}=\langle S ; \cdot\rangle$ назьваются инверснымм друг другу, если $a b a=a$ и $b a b=b$. Полугруппа $\mathscr{S}$ называется инверсной, если каждый элемент полугрупшы $\mathscr{S}$ имеет в $\mathscr{S}$ единственньй инверсньй к нему элемент.

Взаимно однозначным частичным преобразованием множества $X$ будем называть любое взаимно однозначное отображение $\alpha$ некоторого подмножества $Y$ множества $X$ на подмножество $\alpha(Y)$ множества $X$. Через $\alpha^{-1}$ обозначим отображение множества $\alpha(Y)$ на множество $Y$, обратное к $\alpha$. Пусть $S_{X}-$ совокупность всех взаимно однозначных частичных преобразований множества $X$, включая отображение пустого подмножества $\varnothing$ на себя (обозначим последнее как $\varnothing$ ). Произведение $\alpha \cdot \beta$ элементов из $S_{X}$ определим следующим образом: если $\operatorname{dom} \alpha \cap \operatorname{rang} \beta=\varnothing$, то $\alpha \cdot \beta=\varnothing$, иначе пусть $Y=\operatorname{dom} \alpha \cap \operatorname{rang} \beta$ и $\alpha \cdot \beta$ полагаем равньм суперпозиции отображений $\beta \mid \beta^{-1}(\operatorname{dom} \alpha \cap \operatorname{rang} \beta)$ и $\alpha \mid \operatorname{dom} \alpha \cap \operatorname{rang} \beta$. Очевидно, что $\alpha \cdot \beta \in S_{X}$. Непосредственно замечается, что полугруппа $\mathscr{S}_{X}=\left\langle S_{X} ; \cdot\right\rangle$ является инверсной $\left(\alpha^{-1}\right.$ - единственный инверсньй к $\alpha$ элемент из $\left.S_{X}\right)$.

В. В. Вагнером и Г. Престоном доказано следующее утверждение о представлении инверсных полугрупп.

ТЕОРема 1.12 [12], [64], [59]. Произвольная инверсная полугруппа изоморфна инверсной подполугруппе инверсной полугруппь $\mathscr{S}_{X}=\left\langle S_{X} ; \cdot\right\rangle$ для некоторого мноэества $X$.

\section{Глава 2.}

Условные термы и условные многообразия

1. Условные термы и условно термальные функции. Для удобства обозначений договоримся через $={ }^{1}$ обозначать обычное равенство, а через $=^{0}$ - его отрицание. 
ОПРЕДЕЛЕНИЕ 2.1. Условием сигнатуры $\sigma$ будем назьвать конечную совокупность $\mathscr{T}(\bar{x})$ равенств и неравенств вида

$$
\mathscr{T}(\bar{x})=\left\{\begin{array}{c}
t_{1}^{1}(\bar{x})={ }^{i_{1}} t_{1}^{2}(\bar{x}) \\
\ldots \ldots \ldots \ldots \cdots \cdots \\
t_{n}^{1}(\bar{x})={ }^{i_{n}} t_{n}^{2}(\bar{x})
\end{array},\right.
$$

где $t_{j}^{i}(\bar{x})$ - термы сигнатуры $\sigma$, a $i_{j} \in\{0,1\}$. Полной системой условий сигнатуры $\sigma$ будем называть набор $\left\{\mathscr{T}_{1}(\bar{x}), \ldots, \mathscr{T}_{k}(\bar{x})\right\}$ условий данной сигнатуры такой, что формула $\forall \bar{x}\left(\bigvee_{i=1}^{k} \& \mathscr{T}_{i}(\bar{x})\right)$ является тождественно истинной и для различных $l, m \leqslant k$ формулы \& $\mathscr{T}_{l}(\bar{x}) \& \& \mathscr{T}_{m}(\bar{x})$ невыполнимы.

На основе понятия условия индуктивно определим понятие условного терма.

ОПРЕДЕЛЕНИЕ 2.2. Понятие условного терма сигнатуры $\sigma$ определяется индукцией за конечное число шагов с помошњю следуюших правил:

а) любая переменная или константа сигнатуры $\sigma$ является условньм термом;

б) если $t_{1}(\bar{x}), \ldots, t_{n}(\bar{x})$ - условные термы сигнатуры $\sigma$ и $f\left(x_{1}, \ldots, x_{n}\right)$ - фуннциональньй символ, входящий в $\sigma$, то $f\left(t_{1}(\bar{x}), \ldots, t_{n}(\bar{x})\right)$ - также условный терм сигнатуры $\sigma$;

в) если $t_{1}(\bar{x}), \ldots, t_{n}(\bar{x})$ - условные термы сигнатуры $\sigma$, a $\left\{\mathscr{T}_{1}(\bar{x}), \ldots, \mathscr{T}_{n}(\bar{x})\right\}-$ полная система условий этой сигнатуры, то

$$
t(\bar{x})=\left\{\begin{array}{l}
\mathscr{T}_{1}(\bar{x}) \rightarrow t_{1}(\bar{x}) \\
\ldots \ldots \ldots \ldots \\
\mathscr{T}_{n}(\bar{x}) \rightarrow t_{n}(\bar{x})
\end{array}\right.
$$

также является условным термом сигнатуры $\sigma$;

г) любой условньй терм сигнатуры $\sigma$ строится за конечное число шагов по правилам а)-в).

Определим теперь семантику условных термов сигнатуры $\sigma$, т.е. функции на алгебрах данной сигнатуры, вычислимые с помошью условных термов, при этом не будем делать формальных различий в обозначениях условного терма и соответствующей ему функции на алгебре $\mathscr{A}$ сигнатуры $\sigma$. Для правил а), б) из определения условного терма предполагаем стандартные интерпретации, имеюшие место при интерпретации термов сигнатуры $\sigma$ на алгебрах этой сигнатуры. Если же условный терм $t(\bar{x})$ построен по правилу в) определения 2.2 , то для любых элементов $\bar{a}$ из алгебры $\mathscr{A}$ (соответствующих переменным $\bar{x}$ условного терма $t(\bar{x}))$ положим $\mathscr{A} \models t(\bar{a})=b$, если для некоторого $i \leqslant n$ имеет место $\mathscr{A} \models \mathscr{T}_{i}(\bar{a})$ и $\mathscr{A} \models t_{i}(\bar{a})=b$. Функции, определимые на алгебре $\mathscr{A}$ с помошью условных термов, будем называть условно термальнылми функиями.

Стандартным образом, с помощью построения в случае в) подходящего двоичного дерева, замечается, что правило в) определения 2.2 может быть заменено на следующее:

для любых термов $t_{1}(\bar{x}), t_{2}(\bar{x})$ сигнатуры $\sigma$ и любых условных термов $t_{3}(\bar{x}), t_{4}(\bar{x})$ этой сигнатуры

$$
t(\bar{x})=\left\{\begin{array}{l}
t_{1}(\bar{x})=t_{2}(\bar{x}) \rightarrow t_{3}(\bar{x}) \\
t_{1}(\bar{x}) \neq t_{2}(\bar{x}) \rightarrow t_{4}(\bar{x})
\end{array}\right.
$$

также является условным термом сигнатуры $\sigma$. 
При этом классы условно термальных функций на любой алгебре сигнатуры $\sigma$, определенные с помощью определения 2.2 и с помошью указанной вариации правила в) в определении 2.2 , совпадают.

В качестве простейшего естественного, но принципиально важного примера условно термальных функций укажем на дискриминатор $d(x, y, z)$. Действительно, дискриминатор $d(x, y, z)$ определим на универсальной алгебре любой сигнатуры условным термом

$$
t_{d}(x, y, z)=\left\{\begin{array}{l}
x \neq y \rightarrow x \\
x=y \rightarrow z
\end{array} .\right.
$$

Обычные термы сигнатуры $\sigma$ будем далее назьвать стандартными. Любой стандартный терм, в частности, является и условным.

Нетрудно заметить, что, проводя одновременную индукцию в определении понятия условия и условного терма (т.е. допуская в определении условия произвольные конечные системы равенств и неравенств между условными термами), мы, в конечном итоге, получаем тот же класс условно термальных функций на алгебрах, что и с помошюю определения 2.2. Тем самым, действительно, понятие условно термальной функции соответствует понятию функции, вычислимой на алгебре с помощю программы вычислений, составленной из простейших (сигнатурных) программ при помощи оператора суперпозиции и условного оператора.

ОПРЕДЕЛЕНИЕ 2.3. Будем говорить, что условньй терм $t(\bar{x})$ имеет нормальную форму, если $t(\bar{x})$ либо стандартный, либо

$$
t(\bar{x})=\left\{\begin{array}{l}
\mathscr{T}_{1}(\bar{x}) \rightarrow t_{1}(\bar{x}) \\
\cdots \ldots \ldots \ldots \ldots \\
\mathscr{T}_{n}(\bar{x}) \rightarrow t_{n}(\bar{x})
\end{array},\right.
$$

где $t_{i}(\bar{x})$ - стандартные термы рассматриваемой сигнатуры, а $\mathscr{T}_{i}(\bar{x})$ - условия, определенные согласно определению 2.1.

Достаточно очевидно следующее утверждение.

Лемма 2.1 [35]. Для любого условного терма $t(\bar{x})$ сигнатурь $\sigma$ существует условный терм $t^{\prime}(\bar{x})$ этой сигнатуры, имеющ,й нормальную форму и такой, что для любой алгебры $\mathscr{A}$ сигнатуры $\sigma$ условным термам $t(\bar{x})$ и $t^{\prime}(\bar{x})$ соответствует на А⿱ одна и та жсе условно термальная функиия.

Таким образом, работая далее с условными термами, всегда можно предполагать, что они заданы в нормальной форме.

В силу леммы 2.1 условно термальные функции на алгебре суть кусочно термальные. Иначе говоря, если $f\left(x_{1}, \ldots, x_{n}\right)$ - условно термальная функция на алгебре $\mathscr{A}$, то сушествуют разбиение $T_{1}, \ldots, T_{k}(k \in \omega)$ декартовой степени $\mathscr{A}^{n}$ алгебры $\mathscr{A}$, определяемое условиями $\mathscr{T}_{1}(\bar{x}), \ldots, \mathscr{T}_{k}(\bar{x})$ сигнатуры алгебры $\mathscr{A}$, и термы $t_{1}\left(x_{1}, \ldots, x_{n}\right), \ldots$, $t_{k}\left(x_{1}, \ldots, x_{n}\right)$ (стандартные) алгебры $\mathscr{A}$ такие, что функция $f$ на кортежах элементов алгебры $\mathscr{A}$, входяших в $T_{i}(i \leqslant k)$, совпадает с термальной функцией, определяемой термом $t_{i}(\bar{x})$ на $\mathscr{A}$.

Очевидно, что любая условно термальная функция $f\left(x_{1}, \ldots, x_{n}\right)$ на алгебре $\mathscr{A}$ определима на этой алгебре с помошю бескванторной формулы исчисления предикатов 
первого порядка, т.е. сушествует бескванторная формула $\Phi_{f}\left(x_{1}, \ldots, x_{n}, y\right)$ сигнатуры алгебры $\mathscr{A}$ такая, что для любых элементов $\bar{a}, b \in \mathscr{A} \mathscr{A} \models f(\bar{a})=b$ тогда и только тогда, когда $\mathscr{A} \models \Phi_{f}(\bar{a}, b)$. Легко заметить, что обратное неверно, т.е. далеко не любая функция, определенная на универсальной алгебре с помощью бескванторной формулы, является условно термальной.

ОПРЕДЕЛЕНИЕ 2.4. Формулу $\Phi(\bar{x}, y)$ исчисления предикатов первого порядка назовем $y$-функииональной на классе алгебр $\mathscr{K}$, если имеет место $\mathscr{K} \models \forall \bar{x} \exists$ ! $y(\bar{x}, y)$. Формулу $\Phi(\bar{x}, y)$ назовем $y$-зависимой, если любая атомная подформула формулы $\Phi(\bar{x}, y)$, содержашая переменную $y$, имеет вид $y=h(\bar{x})$, где $h(\bar{x})-$ терм, не содержащий $y$, и $y$ не входит в отрицания атомных формул, входяших в $\Phi(\bar{x}, y)$.

Синтаксическое описание условно термальных функций содержится в следующем утверждении.

ТЕОРема 2.1 [36]. Формула $\Phi(\bar{x}, y)$ исчисления предикатов первого порядка определяет условно термальнье функиии на алгебрах некоторого класса К тогда и только тогда, когда на классе $\mathscr{K}$ формула $\Phi(\bar{x}, y)$ эквивалентна некоторой бескванторной у-функциональной, у-зависимой формуле.

Далее, в главе 3, мы укажем чисто алгебраическую характеризацию условно термальных функций на конечных и равномерно локально конечных алгебрах.

Очевидно, что подалгебры универсальных алгебр замкнуты относительно условно термальных функций и вычисление условно термальных функций коммутирует как с переходом к подалгебрам, так и с изоморфизмами алгебр. В то же время, как легко видеть, вычисление условного терма, определяющего дискриминатор, не коммутирует с гомоморфизмами, имеющими ненулевое ядро, и вычисление этого терма на квадрате неодноэлементной алгебры не сводится к вычислению этого терма на проекциях квадрата на сомножитель.

Далее для любой универсальной алгебры $\mathscr{A}$ через $T(\mathscr{A})$ будем обозначать совокупность всех термальных функций на $\mathscr{A}$, через $C T(\mathscr{A})-$ совокупность всех условно термальных функций на $\mathscr{A}$, а через $F(\mathscr{A})$ - совокупность всех функций, определенных на основном множестве алгебры $\mathscr{A}$. Имеют место естественные включения

$$
T(\mathscr{A}) \subseteq C T(\mathscr{A}) \subseteq F(\mathscr{A})
$$

и столь же естествен вопрос, для каких алгебр $\mathscr{A}$ эти включения обращаются в равенства.

Условная термальность дискриминатора на любой алгебре и отмеченная вьше эквивалентность определения 2.2 и его вариации непосредственно дают ответ на вопрос, для каких алгебр $\mathscr{A}$ совокупности термальных и условно термальных функций на $\mathscr{A}$ совпадают.

ЛЕмма 2.2. Равенство $T(\mathscr{A})=C T(\mathscr{A})$ әквивалентно тому, что алгебра $\mathscr{A}$ дискриминаторна.

В дальнейшем для любой универсальной алгебры $\mathscr{A}$ через $\mathscr{A}^{d}$ будем обозначать обогащение алгебры $\mathscr{A}$ путем добавления в ее сигнатуру новой трехместной функции $d(x, y, z)$, интерпретируемой на алгебре $\mathscr{A}^{d}$ с помошю дискриминатора. Из утверждения леммы 2.2 , в частности, следуют равенства $T\left(\mathscr{A}^{d}\right)=C T\left(\mathscr{A}^{d}\right)=C T(\mathscr{A})$. 
Эти равенства позволяют в дальнейшем использовать результаты о дискриминаторных алгебрах и многообразиях в вопросах, связанных с условно термальными функциями на произвольных алгебрах.

Принятое нами ограничение на счетность сигнатуры влечет, что из равенства $C T(\mathscr{A})=F(\mathscr{A})$ вытекает конечность алгебры $\mathscr{A}$.

ОПРЕДЕЛЕНИЕ 2.5. Алгебру $\mathscr{A}$ назовем условно примальной, если $\mathscr{A}$ конечна и любая функция на основном множестве алгебры $\mathscr{A}$ является условно термальной $(C T(\mathscr{A})=F(\mathscr{A}))$. Алгебру $\mathscr{A}$ назовем финитарно аппроксимируемой условными термами, если для любого $n \in \omega$, любого конечного подмножества $B \subseteq \mathscr{A}^{n}$ и любой функции $f: \mathscr{A}^{n} \rightarrow \mathscr{A}$ найдется условньй терм $t(\bar{x})$ алгебры $\mathscr{A}$ от $n$ переменных такой, что ограничения $f \mid B$ и $t \mid B$ функции $f$ и условно термальной функции $t$ на множество $B$ совпадают.

Имеет место следующая простая характеризация условно примальных алгебр и алгебр, финитарно апшроксимируемых условными термами (ср. с характеризацией примальных алгебр в теореме 1.8).

Теорема 2.2 [35]. Алгебра $\mathscr{A}$ финитарно аппроксимируема условными термами тогда и только тогда, когда $\mathscr{A}$ не имеет собственных подалгебр и нетривиальных автоморфизмов. Конечная алгебра $\mathscr{A}$ условно примальна тогда и только тогда, когда $\mathscr{A}$ не имеет собственных подалгебр и нетривиальных автоморфизмов.

Доказательство этой теоремы в [35] влечет, в частности, новое доказательство теоремы 1.8 .

Вернемся к определимости условно термальных функций. К понятию определимости функций на основном множестве универсальной алгебры можно подходить с различных позищий: это и определимость рассматриваемой функции формулами того или иного класса (к примеру, определимость функции формулой исчисления предикатов первого порядка - см. теорему 2.1) и, более конструктивно, определимость функции заданием программы ее вычисления (условно термальные функции), определимость свойств значения функции по свойствам ее аргументов и т. д.

Очевидно, что для любой универсальной алгебры $\mathscr{A}$ сигнатуры $\sigma$ и любого $n \in \omega$ совокупность

$$
\begin{aligned}
T_{n}(\mathscr{A})=\left\{\mathscr{T}\left(\mathscr{A}^{n}\right)=\left\{\bar{a}=\left\langle a_{1}, \ldots, a_{n}\right\rangle \in \mathscr{A}^{n} \mid \mathscr{A} \models \mathscr{T}(\bar{a})\right\} \mid\right. \\
\left.\mathscr{T}(\bar{x})-\text { условие сигнатуры } \sigma \text { от переменных } \bar{x}=\left\langle x_{1}, \ldots, x_{n}\right\rangle\right\}
\end{aligned}
$$

образует базу открыто-замкнутых окрестностей некоторой топологии $\mathscr{T}_{n}(\mathscr{A})$ (вообще говоря, не обязательно $T_{1}$-топологии). Также очевидно, что для любого $n \in \omega$ топология $\mathscr{T}_{n}(\mathscr{A})$ содержит тихоновскую степень $\left(\mathscr{T}_{1}(\mathscr{A})\right)^{n}$ топологии $\mathscr{T}_{1}(\mathscr{A})$. Эти топологии $\mathscr{T}_{n}(\mathscr{A})(n \in \omega)$ будем далее называть условными топологиями на алгебpe $\mathscr{A}$.

Рассмотрение этих топологий позволяет ввести ряд естественных классов функций на универсальных алгебрах.

ОПреДЕЛЕНИЕ 2.6. Функшию $f: \mathscr{A}^{n} \rightarrow \mathscr{A}$ назовем непрерывной на алгебре $\mathscr{A}$, если $f$ есть непрерывное отображение топологического пространства $\mathscr{T}_{n}(\mathscr{A})$ в топологическое пространство $\mathscr{T}_{1}(\mathscr{A})$. Таким образом, $f: \mathscr{A}^{n} \rightarrow \mathscr{A}$ непрерывна на $\mathscr{A}$, если 
для любых $a_{1}, \ldots, a_{n} \in \mathscr{A}$ и любого условия $\mathscr{T}(x)$ такого, что $\mathscr{A} \models \mathscr{T}\left(f\left(a_{1}, \ldots, a_{n}\right)\right)$, найдется условие $\mathscr{T}^{\prime}\left(x_{1}, \ldots, x_{n}\right)$ такое, что $\mathscr{A} \models \mathscr{T}^{\prime}\left(a_{1}, \ldots, a_{n}\right)$ и $\mathscr{A} \models \forall x_{1}, \ldots, x_{n}$ $\left(\mathscr{T}^{\prime}\left(x_{1}, \ldots, x_{n}\right) \rightarrow \mathscr{T}\left(f\left(x_{1}, \ldots, x_{n}\right)\right)\right)$.

Иначе говоря, непрерывность функции $f: \mathscr{A}^{n} \rightarrow \mathscr{A}$ означает локальную определимость диаграммных (описьваемых бескванторными формулами исчисления предикатов первого порядка) свойств значений функции $f$ с помошњю диаграммных свойств ее аргументов.

Естественным образом отказ от локальности в приведенном определении рассматривается как равномерная непрерывность функции на алгебре.

ОПРЕДЕЛЕНИЕ 2.7. Функцию $f: \mathscr{A}^{n} \rightarrow \mathscr{A}$ назовем равномерно непрерывной на алгебре $\mathscr{A}$, если возможна (глобальная) определимость диаграммных свойств значения функции $f$ по диаграммным свойствам ее аргументов. То есть если для любого условия $\mathscr{T}(x)$ найдется дизъюнкция условий $\mathscr{T}_{1}(\bar{x}) \vee \cdots \vee \mathscr{T}_{k}(\bar{x})$ (иначе говоря, бескванторная формула сигнатуры алгебры $\mathscr{A}$ ) от переменных $x_{1}, \ldots, x_{n}$ такая, что

$$
\mathscr{A} \models \forall x_{1}, \ldots, x_{n}\left(\left[\mathscr{T}_{1}(\bar{x}) \vee \cdots \vee \mathscr{T}_{k}(\bar{x})\right] \leftrightarrow \mathscr{T}(f(\bar{x}))\right) .
$$

Через $C F(\mathscr{A})$ обозначим совокупность всех непрерьвных, а через $U C F(\mathscr{A})$ - всех равномерно непрерьвных функций на алгебре $\mathscr{A}$.

Наконец, через $\operatorname{def}(\mathscr{A})$ обозначим совокупность всех функций на $\mathscr{A}$, определимых бескванторными формулами исчисления предикатов первого порядка.

Для любой алгебры $\mathscr{A}$ очевидным образом имеет место следующая диаграмма включений:

$$
T(\mathscr{A}) \subseteq C T(\mathscr{A}) \subseteq U C \stackrel{\subseteq \operatorname{def}(\mathscr{A}) \subseteq}{F(\mathscr{A}) \subseteq C F(\mathscr{A}) \subseteq} \subseteq^{F(\mathscr{A})}
$$

При этом в общем случае все включения здесь собственные (см. [44]).

Вьше уже были описаны алгебры, для которых имеют место равенства $T(\mathscr{A})=$ $C T(\mathscr{A})$ и $C T(\mathscr{A})=F(\mathscr{A})$. Остановимся на условиях вьполнимости других равенств в приведенной диаграмме. Для двух классов функций $\mathscr{K}^{1} \subseteq \mathscr{K}^{2}$ будем говорить, что $\mathscr{K}^{2}$-функции финитарно аппроксимируются $\mathscr{K}^{1}$-функииями на алгебре $\mathscr{A}$ (обозначим это как $\left.\mathscr{K}^{1}={ }_{\omega} \mathscr{K}^{2}\right)$, если для любой $\mathscr{K}^{2}$-функции $f: \mathscr{A}^{n} \rightarrow \mathscr{A}$ и любого конечного подмножества $B \subseteq \mathscr{A}^{m}$ сушествует $\mathscr{K}^{1}$-функция $g: \mathscr{A}^{m} \rightarrow \mathscr{A}$ такая, что $f|B=g| B$. (В теореме 2.2 приведены условия: для каких $\mathscr{A}$ имеет место $C T(\mathscr{A})={ }_{\omega} F(\mathscr{A})$.)

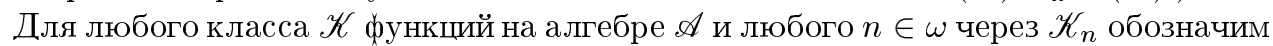
совокупность всех $n$-местных $\mathscr{K}$-функций.

Теорема 2.3 [44]. Для любой алгебры $\mathscr{A}$ следующие условия әквивалентнь:

1) $C T_{1}(\mathscr{A})=U C F_{1}(\mathscr{A})$,

2) $C T(\mathscr{A})=F(\mathscr{A})$,

3) $\mathscr{A}$ не имеет собственных подалгебр и нетривиальных автоморфизмов.

Таким образом, для конечных алгебр А эквивалентны условия:

1) $C T_{1}(\mathscr{A})=U C F_{1}(\mathscr{A})$

2) $C T(\mathscr{A})=F(\mathscr{A})$,

3) $\mathscr{A}$ не имеет собственных подалгебр и нетривиальных автоморфизмов,

4) условные топологии на $\mathscr{A}$ дискретны (в том числе являются $T_{1}$-топологиями) и $\mathscr{A}$ не имеет собственных подалгебр. 
Теорема 2.4 [44]. Для любой алгебры $\mathscr{A}$ следующие условия эквивалентны:

1) $U C F(\mathscr{A})=C F(\mathscr{A})$,

2) условные топологии на алгебре $\mathscr{A}$ компактнь, либо топология $\mathscr{T}_{1}$ на $\mathscr{A}$ тривиальна,

3) топология $\mathscr{T}_{1}$ на $\mathscr{A}$ тривиальна (т.е. для любых а, $b \in \mathscr{A}$ существует изоморфизм подалгебры $\langle a\rangle_{\mathscr{A}}$, порожсденной элементом а, на подалгебру $\langle b\rangle_{\mathscr{A}}$, переводящий а в $\left.b\right)$, либо алгебра $\mathscr{A}$ слабо атомно компактна.

Напомним, что алгебра $\mathscr{A}$ слабо атомно компактна, если для любого $n \in \omega$, любой совокупности бескванторных формул $\left\{\Phi\left(x_{1}, \ldots, x_{n}\right) \mid i \in I\right\}$, конечно вьполнимой на $\mathscr{A}$, имеет место вьполнимость на $\mathscr{A}$ всей этой совокупности в целом.

Достаточно очевидно, что для любой алгебры $\mathscr{A}$ имеет место $U C F(\mathscr{A})={ }_{\omega} C F(\mathscr{A})$.

Теорема 2.5 [44]. а) Для любой алгебры $\mathscr{A}$ следуюшие условия эквивалентнь:

1) $\operatorname{def}(\mathscr{A})=F(\mathscr{A})$,

2) $\mathscr{A}$ конечна, $\mathscr{T}_{1}$-топология на $\mathscr{A}$ дискретна,

3) $\mathscr{A}$ конечна и любые различные однопорожсденные подалгебры алгебры $\mathscr{A}$ не изоморфны.

б) Для любой алгебры $\mathscr{A}$ следующие условия әквивалентны:

1) $\operatorname{def}(\mathscr{A})=\omega F(\mathscr{A})$,

2) $\mathscr{T}_{1}$-топология на $\mathscr{A}$ дискретна,

3) любая однопорожденная подалгебра алгебры $\mathscr{A}$ конечно определима среди подалгебр алгебры $\mathscr{A}$ и любье различнье однопорожсденные подалгебры алгебри $\mathscr{A}$ не изоморфны.

Отсюда, в частности, вытекает следующее утверждение.

СЛЕДСТВИЕ 2.1 [44]. Для любой конечной алгебры А્A следующие условия әквивалентны:

1) $C T(\mathscr{A})=\operatorname{def}(\mathscr{A})$,

2) все подалгебры алгебры $\mathscr{A}$ замкнуты относительно любой функиии, определимой на $\mathscr{A}$ бескванторной формулой.

2. Условные тождества и условные многообразия. По аналогии с понятием тождества введем следующее определение.

ОПРЕДЕЛЕНИЕ 2.8. Под условнылм тождеством будем понимать формальное равенство двух условных термов $t_{1}(\bar{x})=t_{2}(\bar{x})$ (для простоты записи будем считать, что оба условных терма $t_{1}(\bar{x})$ и $t_{2}(\bar{x})$ зависят от одних и тех же переменных, вводя в рассмотрение, если требуется, фиктивные переменные). Условное тождество $t_{1}(\bar{x})=t_{2}(\bar{x})$ истинно на алгебре $\mathscr{A}$ соответствующей сигнатуры, если для любых $\bar{a} \in \mathscr{A}$ имеет место $\mathscr{A} \models t_{1}(\bar{a})=t_{2}(\bar{a})$. Под условным многообразием будем понимать класс всех алгебр фиксированной сигнатуры, на которых истинна некоторая совокупность условных тождеств.

Очевидно, что любое условное многообразие является универсальным классом алгебр (аксиоматизируемо $\forall$-формулами исчисления предикатов первого порядка). Для любого класса $\mathscr{K}$ алгебр некоторой фиксированной сигнатуры $\sigma$ через $\mathscr{M}^{*}(\mathscr{K})$ обозначим наименьшее условное многообразие, содержащее этот класс. Через $P_{u}(\mathscr{K})$ 
$(S(\mathscr{K}))$ будем обозначать класс алгебр, состоящий из любых ультрапроизведений $\mathscr{K}$-алгебр (из любых подалгебр $\mathscr{K}$-алгебр), а через $\mathscr{K}^{+}$- класс всех алгебр, получаемьй добавлением к $\mathscr{K}$ одноэлементной алгебры соответствующей сигнатуры. Имеет место

ТеОРема 2.6 [35]. Для любого класса алгебр $\mathscr{K}$ порожденное им условное многообразие $\mathscr{M}^{*}(\mathscr{K})$ есть наименьший универсальный класс алгебр, включающий в себя класс $\mathscr{K}$ и одноэлементную алгебру соответствующей сигнатурь.

Тем самым, имеет место формула $\mathscr{M}^{*}(\mathscr{K})=\operatorname{ISP}\left(\mathscr{K}^{+}\right)=\left(I S P_{U}(\mathscr{K})\right)^{+}$. В частности, класс алгебр $\mathscr{K}$ является условньм многообразием тогда и только тогда, когда $\mathscr{K}$ - универсальньй класс, включаюший в себя одноэлементую алгебру.

СлЕДСТВИЕ 2.2. Для любой конечной алгебры $\mathscr{A}$ имеет место равенство $\mathscr{M}^{*}(\mathscr{K})=I S(\mathscr{A})^{+}$, т.е. условное многообразие, порохсденное $\mathscr{A}$, состоит из алгебр, изоморфных подалгебрам алгебры $\mathscr{A}$, и одноэлементных алгебр.

Утверждение этого следствия позволяет использовать результаты об условных многообразиях для изучения отдельно взятых конечных алгебр, что невозможно при работе с произвольными традиционными многообразиями. Однако, с другой стороны, незамкнутость универсальных классов относительно гомоморфизмов не позволяет при изучении условных многообразий использовать технику, связанную с решетками конгруэнций (даже относительных, т.е. тех, фактор по которьм лежит в этом же классе). Более того, сушествуют (см. [35]) универсальные алгебры $\mathscr{A}_{1}, \mathscr{A}_{2}$ и условное многообразие $\mathscr{M}$ такие, что:

1) в алгебре $\mathscr{A}_{1}$ имеются элементы $a \neq b$, для которых не существует наименьшей конгруэнции $\Theta \in \operatorname{Con} \mathscr{A}_{1}$ такой, что $\langle a, b\rangle \in \Theta$ и $\mathscr{A}_{1} / \Theta \in \mathscr{M}^{*}\left(\mathscr{A}_{1}\right)$ (в алгебре $\mathscr{A}_{1}$ нет главной $\mathscr{M}^{*}\left(\mathscr{A}_{1}\right)$-конгруэнции, порожденной парой $\left.\langle a, b\rangle\right)$;

2) сушествуют $\Theta_{1}, \Theta_{2} \in \operatorname{Con} \mathscr{A}_{2}, \mathscr{A}_{2} \in \mathscr{M}$, и не сушествует наименьшей конгруэнции $\Theta$ такой, что $\Theta_{1}, \Theta_{2} \leqslant \Theta$ и $\mathscr{A}_{2} / \Theta \in \mathscr{M}$ (совокупность $\mathscr{M}$-конгруэнщий на $\mathscr{A}_{2}$ не является верхней полурешеткой).

Более эффективньм инструментом, чем конгруэнции, при изучении строения алгебр из условных многообразий, как это будет видно из материала следующей главы, являются решетки подалгебр и полугруппы изоморфизмов между подалгебрами алгебр данного условного многообразия.

3. Исчисление условных тождеств. Естественньм представляется вопрос о построении формального исчисления условных тождеств, подобного биркгофовскому исчислению тождеств. Теорема 1.9 позволяет построить подобное исчисление.

Пусть $T$ - некоторая совокупность условных тождеств сигнатуры $\sigma$ и $t_{1}(\bar{x})=t_{2}(\bar{x})$ - еше одно условное тождество этой сигнатуры. Через $T \models t_{1}(\bar{x})=t_{2}(\bar{x})$ обозначим тот факт, что для любой алгебры $\mathscr{A}$, на которой истинны условные тождества из $T$, истинно и условное тождество $t_{1}(\bar{x})=t_{2}(\bar{x})$.

Через $A x_{\sigma}$ (где $\sigma$ - некоторая фиксированная сигнатура) обозначим следующую совокупность условных тождеств:
1) $\left\{\begin{array}{l}x=z \rightarrow z \\ x \neq z \rightarrow x\end{array}=x\right.$,
2) $\left\{\begin{array}{l}x=y \rightarrow x \\ x \neq y \rightarrow x\end{array}=x\right.$, 
3) $\left\{\begin{array}{l}x=z \rightarrow z \\ x \neq x \rightarrow x\end{array}=z\right.$,

4) $\left\{\begin{array}{l}\left(x=\left\{\begin{array}{l}x=y \rightarrow z \\ x \neq y \rightarrow x\end{array}\right) \rightarrow y\right. \\ \left(x \neq\left\{\begin{array}{l}x=y \rightarrow z \\ x \neq y \rightarrow x\end{array}\right) \rightarrow x\right.\end{array}=y\right.$,

5) для любой функции $f\left(x_{1}, \ldots, x_{n}\right)$ из $\sigma$

$$
\left\{\begin{array}{l}
x=y \rightarrow f\left(z_{1}, \ldots, z_{k}\right) \\
x \neq x \rightarrow x
\end{array}=\left\{\begin{array} { l } 
{ x = y \rightarrow f } \\
{ x \neq y \rightarrow x }
\end{array} \left(\left\{\begin{array}{l}
x=y \rightarrow z_{1} \\
x \neq y \rightarrow x
\end{array}, \ldots,\left\{\begin{array}{l}
x=y \rightarrow z_{k} \\
x \neq y \rightarrow x
\end{array}\right),\right.\right.\right.\right.
$$

6) $\left\{\begin{array}{l}x=y \rightarrow\left\{\begin{array}{l}z_{1}=z_{2} \rightarrow z_{3} \\ z_{1} \neq z_{2} \rightarrow z_{1}\end{array}\right. \\ x \neq y \rightarrow x\end{array}\right.$

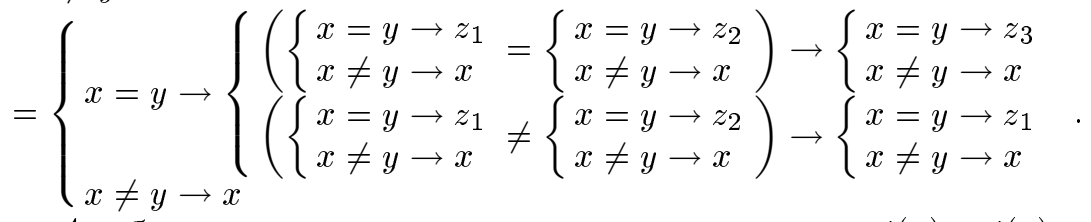

Через $A_{\sigma}$ обозначим совокупность условных тождеств вида $t(\bar{x})=t(\bar{x})$, где $t(\bar{x})-$ произвольный условный терм сигнатуры $\sigma$.

ОПРЕДЕЛЕНИЕ 2.9. Под исчислением условных тождеств сигнатуры б будем понимать совокупность аксиом $A X_{\sigma} \cup A_{\sigma}$ и правил вьвода:

$$
\begin{aligned}
& \frac{t_{1}(\bar{x})=t_{2}(\bar{x})}{t_{2}(\bar{x})=t_{1}(\bar{x})}, \\
& \frac{t_{1}(\bar{x})=t_{2}(\bar{x}), t_{2}(\bar{x})=t_{3}(\bar{x})}{t_{1}(\bar{x})=t_{3}(\bar{x})}, \\
& \frac{q_{1}^{1}(\bar{x})=q_{2}^{1}(\bar{x}), \ldots, q_{1}^{n}(\bar{x})=q_{2}^{n}(\bar{x})}{t_{1}\left(q_{1}(\bar{x}), \ldots, q_{n}(\bar{x})\right)=t_{2}\left(q_{1}(\bar{x}), \ldots, q_{n}(\bar{x})\right)}, \\
& \frac{t_{1}\left(x_{1}, \ldots, x_{n}\right)=t_{2}\left(x_{1}, \ldots, x_{n}\right)}{t_{1}\left(q_{1}(\bar{x}), \ldots, q_{n}(\bar{x})\right)=t_{2}\left(q_{1}(\bar{x}), \ldots, q_{n}(\bar{x})\right)},
\end{aligned}
$$

где $t_{1}(\bar{x}), t_{2}(\bar{x}), t_{3}(\bar{x}), q_{1}(\bar{x}), \ldots, q_{n}(\bar{x}), q_{j}^{i}(\bar{x})$ - произвольные условные термы сигнатуры $\sigma, f\left(x_{1}, \ldots, x_{n}\right) \in \sigma$.

Если $T$ - некоторая совокупность условных тождеств, то под доказательством условного тождества $t_{1}(\bar{x})=t_{2}(\bar{x})$ из совокупности $T$ в исчислении условных тождеств понимаем последовательность

$$
p_{1}^{1}(\bar{x})=p_{2}^{1}(\bar{x}), \ldots, p_{1}^{n}(\bar{x})=p_{2}^{n}(\bar{x})
$$

условных тождеств такую, что условное тождество $p_{1}^{n}(\bar{x})=p_{2}^{n}(\bar{x})$ и есть условное тождество $t_{1}(\bar{x})=t_{2}(\bar{x})$, а для любого $i \leqslant n$ условное тождество $p_{1}^{i}=p_{2}^{i}(\bar{x})$ либо входит в совокупность $A x_{\sigma} \cup A_{\sigma} \cup T$, либо получается из предшествуюших условных тождеств по одному из правил вьвода 1$)-4)$. 
Существование доказательства условного тождества $t_{1}(\bar{x})=t_{2}(\bar{x})$ из совокупности $T$ обозначим как $T \vdash_{c} t_{1}(\bar{x})=t_{2}(\bar{x})$.

Доказательство следующей теоремы полноты для условных тождеств построено на использовании теоремы 1.9, теоремы полноты Биркгофа для обычных тождеств, теоремы 2.6 и того, что для любого универсального класса алгебр $\mathscr{K}$, включающего одноэлементную алгебру, класс $\mathscr{K}^{d}=\left\{\mathscr{A}^{d} \mid \mathscr{A} \in \mathscr{K}\right\}$ является $\mathscr{M}_{S I^{-}}^{+}$классом для некоторого дискриминаторного многообразия $\mathscr{M}$ сигнатуры $\sigma \cup\{d\}$.

ТЕОРема 2.7 [38]. Для любой совокупности тождеств сигнатуры $\sigma$ и любого условного тождества $t_{1}(\bar{x})=t_{2}(\bar{x})$ той жсе сигнатуры отношения $T \models t_{1}(\bar{x})=$ $t_{2}(\bar{x})$ u $T \vdash_{c} t_{1}(\bar{x})=t_{2}(\bar{x})$ равносильньл.

\section{Глава 3.}

\section{Условно рациональная эквивалентность и связанные с ней вопросы}

1. Условно рациональная эквивалентность и категории вложимости. По аналогии с понятием ращиональной эквивалентности введем понятие условной рациональной эквивалентности двух классов универсальных алгебр.

ОПРЕДЕЛЕНИЕ 3.1. Классы алгебр $\mathscr{K}_{1}$ сигнатуры $\sigma_{1}$ и $\mathscr{K}_{2}$ сигнатуры $\sigma_{2}$ условно рационально эквивалентны, если сушествуют отображения $F_{1}\left(F_{2}\right)$ сигнатурных символов операций из $\sigma_{1}\left(\sigma_{2}\right)$ в условные термы сигнатуры $\sigma_{2}\left(\sigma_{1}\right)$ с сохранением арности и при этом:

1) для любой $\mathscr{K}_{1}$-алгебры $\mathscr{A}=\left\langle A ; \sigma_{1}\right\rangle$ алгебра $F_{2}(\mathscr{A})=\left\langle A ; \sigma_{2}\right\rangle$ принадлежит классу $\mathscr{K}_{2}$, здесь $\sigma_{2}$-операции алгебры $F_{2}(\mathscr{A})$ определены $F_{2}\left(\sigma_{2}\right)$-условньми термами алгебры $\mathscr{A}$;

2) для любой $\mathscr{K}_{2}$-алгебры $\mathscr{A}=\left\langle A ; \sigma_{2}\right\rangle$ алгебра $F_{1}(\mathscr{A})=\left\langle A ; \sigma_{1}\right\rangle$ принадлежит классу $\mathscr{K}_{1}$

$3)$ для любой $\mathscr{K}_{1}$-алгебры $\mathscr{A}=\left\langle A ; \sigma_{1}\right\rangle$ имеет место равенство $F_{1}\left(F_{2}(\mathscr{A})\right)=\mathscr{A}$;

4) для любой $\mathscr{K}_{2}$-алгебры $\mathscr{A}=\left\langle A ; \sigma_{2}\right\rangle$ имеет место равенство $F_{2}\left(F_{1}(\mathscr{A})\right)=\mathscr{A}$.

ОПРЕДЕЛЕНИЕ 3.2. Алгебры $\mathscr{A}_{1}, \mathscr{A}_{2}$ условно рационально эквивалентны, если условно рационально эквивалентны классы алгебр $I\left(\left\{\mathscr{A}_{1}\right\}\right)$ и $I\left(\left\{\mathscr{A}_{2}\right\}\right)$, т.е. если существует биекция $\pi$ основного множества алгебры $\mathscr{A}_{1}$ на основное множество алгебры $\mathscr{A}_{2}$ такая, что $C T\left(\mathscr{A}_{1}\right)=C T\left(\pi\left(\mathscr{A}_{2}\right)\right)$. Функции сигнатуры $\sigma_{1}$ определены при этом в алгебре $\pi\left(\mathscr{A}_{1}\right)$ на основном множестве алгебры $\mathscr{A}_{2}$ с помошюю $\pi$-сопряжения функций сигнатуры $\sigma_{1}$ алгебры $\mathscr{A}_{1}$.

Условную рациональную эквивалентность классов $\mathscr{K}_{1}$ и $\mathscr{K}_{2}$ (алгебр $\mathscr{A}_{1}$ и $\mathscr{A}_{2}$ ) обозначим как $\mathscr{K}_{1} \sim_{c} \mathscr{K}_{2}\left(\mathscr{A}_{1} \sim_{c} \mathscr{A}_{2}\right)$.

Через $\overleftrightarrow{\mathscr{K}}$, где $\mathscr{K}$ - некоторьй класс универсальных алгебр, обозначим категорию, объектами которой являются $\mathscr{K}$-алгебры, а морфизмами - изоморфные вложения $\mathscr{K}$-алгебр друг в друга и эпиморфизмы $\mathscr{K}$-алгебр на одноэлементную алгебру соответствующей сигнатуры, если последняя входит в $\mathscr{K}$. Категорию $\overleftrightarrow{\mathscr{K}}$ будем называть категорией вложимлости для класса $\mathscr{K}$. Через $S \underset{\mathscr{K}}{\longrightarrow}$ обозначим стираюший функтор из категории $\overrightarrow{\mathscr{K}}$ в категорию множеств Set. 
ОПРЕДЕЛЕНИЕ 3.3. Классы алгебр $\mathscr{K}_{1}$ и $\mathscr{K}_{2}$ назовем әквивалентнылми по вложимости, если существует изоморфизм $G$ категории $\overrightarrow{\mathscr{K}}_{1}$ на категорию $\overrightarrow{\mathscr{K}}_{2}$, коммутирующий со стирающими функторами этих категорий, т.е. такой, что

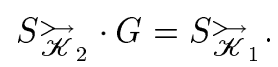

Эквивалентность по вложимости классов $\mathscr{K}_{1}$ и $\mathscr{K}_{2}$ будем обозначать как $\mathscr{K}_{1} \simeq \mathscr{K}_{2}$. Напомним, что $\mathscr{K}_{1} \sim \mathscr{K}_{2}$ означает рациональную эквивалентность этих классов, а $\mathscr{K}_{1} \simeq \mathscr{K}_{2}-$ их эквивалентность (см. определение 1.1).

Имеет место следуюший аналог теоремы А.И. Мальцева о рационально эквивалентных многообразиях.

ТеОРема 3.1 [38]. Для любых условных многообразий $\mathscr{K}_{1}$ и $\mathscr{K}_{2}$ универсальньх алгебр следующие условия эквивалентны:

1) $\mathscr{K}_{1} \sim_{c} \mathscr{K}_{2}$,

2) $\mathscr{K}_{1} \simeq \mathscr{K}_{2}$

3) $\left(\mathscr{K}_{1}\right)^{d} \simeq e\left(\mathscr{K}_{2}\right)^{d}$,

4) $\left(\mathscr{K}_{1}\right)^{d} \simeq\left(\mathscr{K}_{2}\right)^{d}$,

5) $\mathscr{M}\left(\left(\mathscr{K}_{1}\right)^{d}\right) \simeq \mathscr{M}\left(\left(\mathscr{K}_{2}\right)^{d}\right)$,

6) $\mathscr{M}\left(\left(\mathscr{K}_{1}\right)^{d}\right) \sim \mathscr{M}\left(\left(\mathscr{K}_{2}\right)^{d}\right)$,

7) $\mathscr{F}_{\mathscr{M}\left(\left(\mathscr{K}_{1}\right)^{d}\right)}\left(\aleph_{0}\right) \simeq \mathscr{F}_{\mathscr{M}\left(\left(\mathscr{K}_{2}\right)^{d}\right)}\left(\aleph_{0}\right)$.

В доказательстве этой теоремы используются теорема 1.1 (Мальцева о рационально эквивалентных многообразиях), теорема 1.10 (о представимости алгебр дискриминаторного многообразия $\mathscr{M}$ булевыми произведениями $\mathscr{M}_{S}^{+}$-алгебр) и равенства $\mathscr{M}\left(\left(\mathscr{K}_{i}\right)^{d}\right)_{S I}^{+}=\left(\mathscr{K}_{i}^{+}\right)^{d}$.

Утверждение этой теоремы имеет целый ряд как непосредственных, так и более отдаленных следствий, связанных с понятием условного терма.

ОПРЕДЕЛЕНИЕ 3.4. Полугруппой Iso $\mathscr{A}$ внутренних изоморфизмов алгебры $\mathscr{A}$ назовем множество всех изоморфизмов между подалгебрами алгебры $\mathscr{A}$ с добавленным к нему пустьм отображением $\varnothing$, на котором полугрупповая операция определена так же, как в определении полугрупшы $\mathscr{I}_{X}$ (полугруппы взаимно однозначных частичных преобразований множества $X$ ) из п. 4 главы 1.

Будем говорить, что совокупности условно термальных функщий $C T\left(\mathscr{A}_{1}\right)$ и $C T\left(\mathscr{A}_{2}\right)$ алгебр $\mathscr{A}_{1}$ и $\mathscr{A}_{2}$ сопряженш биекцией $\pi$ основного множества алгебры $\mathscr{A}_{1}$ на основное множество алгебры $\mathscr{A}_{2}$, если $C T\left(\mathscr{A}_{1}\right)=\pi\left(C T\left(\mathscr{A}_{2}\right)\right)=\left\{\pi^{-1} f\left(\pi\left(x_{1}\right), \ldots, \pi\left(x_{n}\right)\right)\right.$ $\left.f\left(x_{1}, \ldots, x_{n}\right) \in C T\left(\mathscr{A}_{2}\right)\right\}$.

СЛЕДСТВИЕ 3.1 [38]. ДЛя любих двух конечных алгебр $\mathscr{A}_{1}$ и $\mathscr{A}_{2}$ совокупности $C T\left(\mathscr{A}_{1}\right)$ и $C T\left(\mathscr{A}_{2}\right)$ сопряжень биекиией $\pi$ основного множества алгебры $\mathscr{A}_{1}$ на основное множсество алгебры $\mathscr{A}_{2}$ тогда и только тогда, когда $\pi$ сопрягает полугруппь внутренних изоморфизмов этих алгебр Iso $\mathscr{A}_{1}$ u Iso $\mathscr{A}_{2}$. В частности, если алгебры $\mathscr{A}_{1}$ и $\mathscr{A}_{2}$ определены на одном множестве, то $C T\left(\mathscr{A}_{1}\right)=C T\left(\mathscr{A}_{2}\right)$ тогда и только тогда, когда Iso $\mathscr{A}_{1}=$ Iso $\mathscr{A}_{2}$.

Утверждение теоремы 3.1 позволяет получить алгебраическое описание условно термальных функций на конечных и равномерно локально конечных алгебрах. 
ОПРЕДЕЛЕНИЕ 3.5. Алгебра $\mathscr{A}$ назьвается равномерно локально конечной, если сушествует функция $f: \omega \rightarrow \omega$ такая, что для любых $a_{1}, \ldots, a_{n} \in \mathscr{A}$ мошность подалгебры $\left\langle a_{1}, \ldots, a_{n}\right\rangle \mathscr{A}$ алгебры $\mathscr{A}$, порожденной множеством $\left\{a_{1}, \ldots, a_{n}\right\}$, не превосходит $f(n)$.

ОПРЕДЕЛЕНИЕ 3.6. Функция $g\left(x_{1}, \ldots, x_{n}\right)$, заданная на основном множестве алгебры $\mathscr{A}$, сохраняется внутренними изоморфизмами алгебры $\mathscr{A}$, если:

1) для любых $a_{1}, \ldots, a_{n} \in \mathscr{A}$ имеет место включение $g\left(a_{1}, \ldots, a_{n}\right) \in\left\langle a_{1}, \ldots, a_{n}\right\rangle_{\mathscr{A}}$;

2) для любого изоморфизма $h$ некоторой подалгебры $\mathscr{A}_{1}$ алгебры $\mathscr{A}$ на подалгебру $h\left(\mathscr{A}_{1}\right)$ и для любых $a_{1}, \ldots, a_{n} \in \mathscr{A}_{1}$ имеет место равенство

$$
g\left(h\left(a_{1}\right), \ldots, h\left(a_{n}\right)\right)=h\left(g\left(a_{1}, \ldots, a_{n}\right)\right) .
$$

ТЕОРема 3.2 [36]. Если $\mathscr{A}$ - конечная или равномерно локально конечная алгебра конечной сигнатурь, то условно термальнье функции алгебры $\mathscr{A}$ суть те, которые сохраняются внутренними изоморфизмами алгебры $\mathscr{A}$.

Отметим, что как условие равномерной локальной конечности, так и условие конечности сигнатуры алгебры сушественны в формулировке теоремы 3.2 .

2. Сколемовские и условно термальные функции. Пусть $T$-некоторая теория исчисления предикатов первого порядка функциональной сигнатуры $\sigma$ и $\Phi$ - некоторое предложение этого исчисления. Через $\Phi \frac{*}{f}$ обозначим сколемизацию предложения $\Phi$ с помошью некоторого набора $\bar{f}=\left\langle f_{1}, \ldots, f_{n}\right\rangle$ сколемовских функций (соответствующее определение см., к примеру, в [10]).

ОПРЕДЕЛЕНИЕ 3.7. БУдем ГОворить, что Т однозначно определяет сколемовские функиии предложения $\Phi$, если имеет место

$$
T, \Phi_{f}^{*}, \Phi_{\bar{g}}^{*} \vdash f_{1}=g_{1} \& \cdots \& f_{n}=g_{n},
$$

т.е., действительно, на всех $T$-алгебрах, на которых истинно предложение $\Phi$, набор соответствуюших сколемовских функций определен однозначно.

Заметим, что в силу теоремы Бэта об определимости (см., к примеру, [4], [10]) если $T$ однозначно определяет сколемовские функции предложения $\Phi$, то эти функции определимы формулами исчисления первого порядка сигнатуры $\sigma$ на классе всех $T \cup\{\Phi\}-$ алгебр. При некоторых дополнительных условиях можно утверждать условную термальность этих сколемовских функций.

ТеОРема 3.3 [53]. Пусть $T$ - универсальная теория сигнатуры $\sigma, \Phi-$ предложение этой сигнатурьи и

1) $T \vdash \Phi$,

2) $Т$ однозначно определяет сколемовские функиии $\bar{f}=\left\langle f_{1}, \ldots, f_{n}\right\rangle$ для $\Phi$,

3) для любой $T$-алгебры $\mathcal{A}$, любого $i \leqslant n$, любого кортежа $\bar{a}$ (соответствующей длины) әлементов из $\mathscr{A}$ имеет место включение $f_{i}(\bar{a}) \in\langle\bar{a}\rangle_{\mathscr{A}}$.

Тогда существуют условные термы $t_{1}, \ldots, t_{n}$ сигнатуры $\sigma$, определяющие сколемовские функции $f_{1}, \ldots, f_{n}$ на классе $T$-алгебр.

Условия теоремы 3.3 допускают некоторые вариации. 
СлЕДСТВИЕ 3.2 [53]. Пусть $T$ - универсальная теория сигнатуры $\sigma, \Phi-\forall \exists$ предложсние этой сигнатуры и

1) $T \vdash \Phi$,

2) $Т$ однозначно определяет сколемовские функиии $\bar{f}=\left\langle f_{1}, \ldots, f_{n}\right\rangle$ для Ф.

Тогда существуют условнье термы $t_{1}, \ldots, t_{n}$ сигнатуры $\sigma$, определяющие сколемовские функиии $f_{1}, \ldots, f_{n}$ для $\Phi$ на классе $T$-алгебр.

Требование на $\Phi$ быть $\forall \exists$-предложением в условии следствия 3.2 может быть заменено требованием модельной полноты теории $T$.

\section{3. Морита-эквивалентность по вложимости.}

ОПреДЕлЕниЕ 3.8. Два класса $\mathscr{K}_{1}$ и $\mathscr{K}_{2}$ универсальных алгебр назовем Морита-эквивалентными по вложимости, если категории $\overrightarrow{\mathscr{K}}_{1}$ и $\overrightarrow{\mathscr{K}}_{2}$ изоморфны.

Подобно указанному вьше (теорема 3.1 ) аналогу для условных многообразий теоремы Мальцева о рациональной эквивалентности, теорема Мак-Кензи о Морита-эквивалентных многообразиях (теорема 1.3) имеет следуюший аналог для Морита-эквивалентных по вложимости условных многообразий.

Теорема 3.4 [38]. Два условных многообразия $\mathscr{K}_{1}$ и $\mathscr{K}_{2}$ Морита-эквивалентны по вложимости тогда и только тогда, когда для некоторого натурального $n$ и некоторого обратимого идемпотентного на $\mathscr{K}_{1}^{[n]}$ условного терма $\eta(x)$ сигнатуры класса $\mathscr{K}_{1}^{[n]}$ классы алгебр $\mathscr{K}_{1}^{[n]}(\eta)$ и $\mathscr{K}_{2}$ условно рачионально әквивалентны.

Через Iso* $\mathscr{A}$ обозначим инверсную полугруппу Iso $\mathscr{A}$ внутренних изоморфизмов алгебры $\mathscr{A}$ с выделенным множеством $\left\{\operatorname{id}_{\mathscr{A}_{1}} \mid \mathscr{A}_{1}\right.$ - одноэлементная подалгебра алгебры $\mathscr{A}\}$. Здесь $\mathrm{id}_{X}$ - тождественное отображение множества $X$ на себя. В дальнейшем изоморфизм полугрупп Iso $\mathscr{A}_{1}$ и Iso $\mathscr{A}_{2}$ предполагает изоморфизм полугрупп Iso $\mathscr{A}_{1}$ и Iso $\mathscr{A}_{2}$, coхраняюший выделенные подмножества.

ОПРЕДЕЛЕНИЕ 3.9. Алгебры $\mathscr{A}_{1}$ и $\mathscr{A}_{2}$ назовем схо жими, если для некоторого натурального $n$ и некоторого обратимого идемпотентного условного терма $\eta(x)$ сигнатуры алгебры $\mathscr{A}_{1}^{[n]}$ алгебры $\mathscr{A}_{1}^{[n]}(\eta)$ и $\mathscr{A}_{2}$ условно рационально эквивалентны.

СлЕДСТВИЕ 3.3 [38]. Конечные алгебры $\mathscr{A}_{1}$ и $\mathscr{A}_{2}$ схожи тогда и только тогда, когда полугруппьи Iso* $\mathscr{A}_{1}$ u Iso $^{*} \mathscr{A}_{2}$ изоморфныл.

4. Определимость алгебр производными структурами. K классическим вопросам универсальной алгебры, а также теорий конкретных классов алгебр: груп, решеток, колец и т. д., относится вопрос об определимости алгебр данного рассматриваемого класса $\mathscr{K}$ с помощью тех или иных производных структур (решетки подалгебр, группы автоморфизмов, полугрупш эндоморфизмов и т. д.). Пусть для универсальной алгебры $\mathscr{A}$ через $\mathscr{A}^{\prime}$ будет обозначена некоторая производная от $\mathscr{A}$ алгебраическая система (к примеру, $\mathscr{A}^{\prime}=\operatorname{Sub} \mathscr{A}, \mathscr{A}^{\prime}=\operatorname{Aut} \mathscr{A}, \mathscr{A}^{\prime}=\operatorname{Con} \mathscr{A}, \mathscr{A}^{\prime}=$ Iso $\mathscr{A}$ и т.д.). Тогда указанный вопрос формулируется следуюшим образом: верно ли, что для данного класса $\mathscr{K}$ универсальных алгебр из изоморфизма алгебраических систем $\mathscr{A}_{1}^{\prime}$ и $\mathscr{A}_{2}^{\prime}$ $\left(\mathscr{A}_{1}, \mathscr{A}_{2} \in \mathscr{K}\right)$ вытекает изоморфизм (или иная форма близости) самих $\mathscr{K}$-алгебр $\mathscr{A}_{1}$ и $\mathscr{A}_{2}$ ? Вариацией этого вопроса является и следуюший: описать те $\mathscr{K}$-алгебры $\mathscr{A}$, для которых изоморфизм $\mathscr{A}^{\prime} \cong \mathscr{A}_{1}^{\prime}$ влечет изоморфизм (или иную близость) алгебр 
$\mathscr{A}_{\text {и }} \mathscr{A}_{1}$ для любой $\mathscr{K}$-алгебры $\mathscr{A}_{1} . \mathrm{K}$ примеру, в вопросах, связанных с определимостью решеток решетками своих подрешеток, речь может идти о подобной определимости с точностью до двойственности (так как если решетки $L_{1}$ и $L_{2}$ двойственны, то $\left.\operatorname{Sub} L_{1} \cong \operatorname{Sub} L_{2}\right)$.

Эта ситуация отражена в следуюших определениях.

ОпрЕДЕлЕниЕ 3.10. Для любого класса $\mathscr{K}$ универсальных алгебр конечной сигнатуры $\sigma=\left\langle f_{1}^{n_{1}}, \ldots, f_{k}^{n_{k}}\right\rangle$ и любой последовательности $\bar{t}=\left\langle t_{1}\left(\bar{x}_{1}\right), \ldots, t_{k}\left(\bar{x}_{k}\right)\right\rangle$ условных термов сигнатуры $\sigma$ таких, что $t_{i}\left(\bar{x}_{i}\right)$ зависит от переменных $x_{1}, \ldots, x_{n_{i}}$, для любой $\mathscr{K}$-алгебры $\mathscr{A}=\langle A ; \sigma\rangle$ через $\mathscr{A}^{\bar{t}}$ обозначим алгебру $\mathscr{A}^{\bar{t}}=\left\langle A ; t_{1}, \ldots, t_{k}\right\rangle$, где функции $f_{i}$ сигнатуры $\sigma$ алгебры $\mathscr{A}^{\bar{t}}$ определяются как соответствуюшие условно термальные функции $t_{i}$ алгебры $\mathscr{A}$. Алгебру $\mathscr{A}^{\bar{t}}$ назовем $\bar{t}$-сопряженной $\kappa$ алгебре $\mathscr{A}$.

ОПРЕДЕЛЕНИЕ 3.11. Пусть $\mathscr{A}^{\prime}$ - некоторая (вполне определенная) производная алгебраическая система, соответствуюшая $\mathscr{K}$-алгебре $\mathscr{A}$. Скажем, что производная ' определяет $\mathscr{K}$-алгебру $\mathscr{A}$ в классе $\mathscr{K}$ с точностью до финитарного сопряжения, если сушествует конечное число кортежей $\bar{t}^{1}, \ldots, \bar{t}^{p}$ термов сигнатуры $\sigma$ такое, что для любой алгебры $\mathscr{A}_{1} \in \mathscr{K}$ изоморфизм систем $\mathscr{A}^{\prime} \cong \mathscr{A}_{1}^{\prime}$ влечет существование (хотя бы) одного из следуюших изоморфизмов: $\mathscr{A}_{1} \cong \mathscr{A}^{t^{1}}, \ldots, \mathscr{A}_{1} \cong \mathscr{A}^{p}$. В этом случае скажем также, что производная' определяет $\mathscr{K}$-алгебру $\mathscr{A}$ с точностью до сопряженностей $\bar{t}^{1}, \ldots, \bar{t}^{p}$.

Таким образом, определимость алгебры производной ' с точностью до изоморфизма или определимость решетки с точностью до двойственности - это частные случаи определения 3.11 .

Напомним, что алгебра $\mathscr{A}$ назьвается идемпотентной, если для любой ее сигнатурной функции $f\left(x_{1}, \ldots, x_{n}\right)$ имеет место $\mathscr{A} \models \forall x(f(x, \ldots, x)=x)$. Напомним также, что подкласс $\mathscr{K}_{1}$ класса $\mathscr{K}$ универсальных алгебр назьвается аксиоматизируемым (конечно аксиоматизируемым) внутри класса $\mathscr{K}$, если существует совокупность (конечная совокупность) $T$ формул исчисления предикатов первого порядка такая, что $\mathscr{K}_{1}=\{\mathscr{A} \in \mathscr{K} \mid \mathscr{A} \models T\}$.

Для произвольного класса $\mathscr{K}$ через $\mathscr{K}_{\text {fin }}$ обозначим его подкласс, состояший из всех конечных $\mathscr{K}$-алгебр. В связи с вопросом об описании определимых производной ' $\mathscr{K}$-алгебр полезньм представляется принципиальное решение вопроса об аксиоматизируемости класса '-определимых алгебр внутри класса $\mathscr{K}$. Ниже формулируется решение этого вопроса при некоторых дополнительных условиях, вытекающее из следствия 3.1.

Для класса алгебр $\mathscr{K}$ рассмотрим следуюшее условие на $\mathscr{K}_{\text {fin }}$-класс:

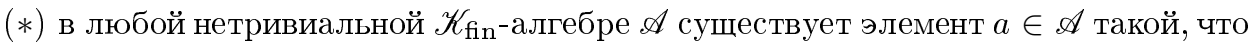
для любого $b \in \mathscr{A}$ подалгебра $\langle a, b\rangle \mathscr{A}$ алгебры $\mathscr{A}$, порожденная парой $\{a, b\}$, не более чем двухэлементна.

Этим свойством, в частности, обладает любой класс полурешеток, решеток и т. д.

ТЕОРема 3.5 [45]. Пусть $\mathscr{K}$ - некоторый конечно аксиоматизируемый класс идемпотентных алгебр конечной сигнатуры $\sigma$, удовлетворяющий условию (*). Пусть $\bar{t}^{1}, \ldots, \bar{t}^{p}$-некоторая совокупность кортежей термов сигнатуры $\sigma$. Тогда класс всех конечных $\mathscr{K}$-алгебр, определимых в $\mathscr{K}$ производной Isо с точностью до сопряженностей $\bar{t}^{1}, \ldots, \bar{t}^{p}$, аксиоматизируем внутри класса $\mathscr{K}_{\mathrm{fin}}$. 
СЛЕДСТВИЕ 3.4 [45]. В условиях теоремы 3.5 если $n$-максимальная арность функиий из $\sigma$ и $\mathscr{F} \mathscr{M}(\mathscr{K})^{(n)}$ конечна, то класс конечных $\mathscr{K}$-алгебр, определимых производной Isо с точностью до сопряженностей $\bar{t}^{1}, \ldots, \bar{t}^{p}$, конечно аксиоматизируем внутри класса $\mathscr{K}_{\text {fin }}$.

\section{Глава 4. \\ Инварианты отношений условной рациональной эквивалентности и схожести}

1. Инварианты отношения условной рациональной эквивалентности конечных алгебр. В силу утверждения следствия 3.1 полугруппа Iso $\mathscr{A}$ играет роль инварианта для классов условно рационально эквивалентных конечных алгебр. В связи с этим представляет интерес описание этой системы инвариантов, т.е. описание подполугрупп $\mathscr{I}$ полугрупा $\mathscr{I}_{A}$ частичных преобразований множеств $A$ таких, что для некоторой алгебры $\mathscr{A}=\langle A ; \sigma\rangle$ имеет место равенство $\mathscr{I}=$ Iso $\mathscr{A}$. Этот вопрос решен в работе Д. А. Бредихина [6]. Аналогичный вопрос для решеток подалгебр и групा автоморфизмов универсальных алгебр решен в работе М. Стоуна [63]. Ниже излагается решение этого вопроса в более удобных для практической работы, чем в работе [6], терминах пар $\langle H ; S\rangle$ - систем $H$ основных множеств подалгебр рассматриваемой алгебры и совокупностей $S$ изоморфизмов между этими подалгебрами.

Говоря, что совокупность $H$ подмножеств некоторого множества $A$ образует peшетку, будем иметь в виду, что $H$ является решеткой относительно порядка, определенного как теоретико-множественное отношение быть подмножеством, при этом решеточная операция $\wedge$ на элементах $H$ является теоретико-множественным пересечением этих элементов как подмножеств множества $A$ (в то время как решеточная операция $\vee$ не обязана быть теоретико-множественной операцией объединения) и супремум, направленный вверх системы подмножеств из $H$, совпадает с теоретико-множественным объединением этой системы.

ОПРЕДЕЛЕНИЕ 4.1. Пусть $H$ - некоторая алгебраическая решетка подмножеств множества $A$, включаюшая в себя и само $A, S$ - некоторая совокупность взаимно однозначных отображений между множествами из $H$.

а) Принципом обратимости для $S$ назовем следующее ее свойство: для любого $g \in S$ отображение $g^{-1}$ также входит в $S$.

б) Принципом композииии для $S$ назовем свойство $S$ : для любых $g, h \in S$ если rang $h=\operatorname{dom} g$, то $h g \in S$.

в) Принципом неподвижных точек для $S$ и $H$ назовем следующее свойство: для любого $g \in S$ множество Fix $g=\{a \in A \mid g(a)=a\}$ входит в $H$.

г) Приниипом ограничения для $S$ и $H$ назовем свойство: для любых $g \in S$ и $C \in H$ если $C \subseteq \operatorname{dom} g$, то $g \mid C \in S$.

д) Приниипом согласованности $S$ и $H$ назовем следующее свойство: для любого $C \in H$ имеет место включение $\mathrm{id}_{C} \in S$, для любого $g \in S$ имеют место включения $\operatorname{dom} g, \operatorname{rang} g \in H$.

е) Принципом глобализаиии назовем следуюшее свойство: для любого $C \in H$ и любого отображения $F: P_{\omega}(C) \rightarrow S$ такого, что

1) для $D \in P_{\omega}(C) \operatorname{dom} F(D)=H(D)$, 
2) для любых $D_{1}, D_{2} \in P_{\omega}(C)$

$$
F\left(D_{1}\right)\left|H\left(D_{1}\right) \cap H\left(D_{2}\right)=F\left(D_{2}\right)\right| H\left(D_{1}\right) \cap H\left(D_{2}\right),
$$

сушествует $h \in S$ такое, что $\operatorname{dom} h=C$ и для любого $D \in P_{\omega}(C) h \mid H(D)=$ $F(D)$. Здесь $P_{\omega}(C)$ - совокупность всех конечных подмножеств множества $C$, а $H(D)$ - наименьшее подмножество из $H$, включаюшее подмножество $D$.

ж) Принципом одноэлементных подалгебр назовем свойство: для любых $a, b \in A$ если $\{a\},\{b\} \in H$, то сушествует $h \in S$ такое, что $\operatorname{dom} h=\{a\}, \operatorname{rang} h=\{b\}$.

Теорема 4.1 [38]. Для любой алгебраической решетки $H$ подмножеств множества $A$, включающей в себя само $A$, и любой системь $S$ биекций подмножеств из $Н$ друг на друга если $S$ и $Н$ удовлетворяют приниипам, перечисленньм в определении 4.1, то существует универсальная алгебра $\mathscr{A}$, определенная на основном множестве $A$, такая, что $H$ есть система основных множеств подалгебр алгебры $\mathscr{A}$ и $S=$ Iso $\mathscr{A}$. Верно и обратное: для любой алгебры $\mathscr{A}$ пара $\langle\mathrm{Sub} \mathscr{A}$, Iso $\mathscr{A}\rangle$ удовлетворяет всем приниипам, перечисленным в определении 4.1.

Теорема 4.1 наряду со следствием 3.1 влечет следующее утверждение.

СЛЕДСТВИЕ 4.1 [38]. Парьц $\langle H ; S\rangle$, состоящие из решетки $H$ подмножеств некоторого конечного мноэсества $A$, включающей в себя само $A$, а также системь биекиий подмножеств из $Н$ друг на друга, удовлетворяющие приниипам обратимости, композиции, неподвижных точек, ограничения, согласованности и приниипу одноэлементных подалгебр, - являются инвариантами классов условно рачионально эквивалентных алгебр.

2. Инварианты отношения условной рациональной эквивалентности йонссоновских локально конечных многообразий. Приведем еще одно теоретико-множественное описание инвариантов отношения условной рациональной эквивалентности некоторого класса условных многообразий, а не отдельных конечных алгебр.

На основе известного определения йонссоновского класса алгебраических систем (см., к примеру, [10]) дадим следуюшее определение.

ОПРЕДЕЛЕНИЕ 4.2. Условное многообразие $\mathscr{K}$ универсальных алгебр назовем йонссоновским, если для $\mathscr{K}$ вьполнены следуюшие условия:

а) для любых конечных алгебр $\mathscr{A}, \mathscr{B} \in \mathscr{K}$ существует конечная алгебра $\mathscr{C} \in \mathscr{K}$ и вложения $f$ и $g$ алгебр $\mathscr{A}$ и $\mathscr{B}$ в алгебру $\mathscr{C}$;

б) для любых конечных алгебр $\mathscr{A}, \mathscr{B}, \mathscr{C} \in \mathscr{K}$ и любых вложений $f, g$ алгебры $\mathscr{C}$ в алгебры $\mathscr{A}$ и $\mathscr{B}$ соответственно сушествуют вложения $f_{1}$ и $g_{1}$ алгебр $\mathscr{A}$ и $\mathscr{B}$ в некоторую конечную $\mathscr{K}$-алгебру $D$ такие, что $f_{1} f=g_{1} g$ (свойство амальгамирования);

в) класс $\mathscr{K}$ содержит бесконечные алгебры;

г) число типов изоморфизма конечных $\mathscr{K}$-алгебр не более чем счетно.

Заметим, что ввиду замкнутости условных многообразий относительно ультрапроизведений любое локально конечное условное многообразие равномерно локально конечно, т.е. сушествует функция $f: \omega \rightarrow \omega$ такая, что любая $n$-порожденная $\mathscr{K}$-алгебра $\mathscr{A}(n \in \omega)$ удовлетворяет неравенству $|\mathscr{A}| \leqslant f(n)$. 
Если локально конечное условное многообразие является йонссоновским, то по известной теореме Морли-Воота (см., к примеру, [10], [19], [30]) существует счетная $\mathscr{K}$-алгебра, являющаяся счетно-универсально-гомогенной для класса $\mathscr{K}$, и такая $\mathscr{K}$-алгебра единственна с точностью до изоморфизма. Обозначим эту алгебру $U_{\mathscr{K}}\left(\aleph_{0}\right)$.

В качестве примера локально конечного йонссоновского многообразия укажем на многообразие булевых алгебр. Счетная безатомная булева алгебра играет роль счетно-универсально-гомогенной алгебры в этом случае.

Пусть $H$ и $G$ - две решетки подмножеств множеств $C$ и $D$ соответственно и для каждого $A \in H$ определено некоторое вложение $f_{A}$ множества $A$ в какое-либо множество из $G$. Если при этом для любых $A_{1}, A_{2} \in H$ из включения $A_{1} \subseteq A_{2}$ следует равенство $f_{A_{2}} \mid A_{1}=f_{A_{1}}$, то совокупность $\mathscr{I}=\left\{f_{A} \mid A \in H\right\}$ назовем решеткой вложений решетки подмножеств $H$ в решетку подмножеств $G$. Отображение $g_{\mathscr{I}}$, определенное как $\bigcup_{A \in H} f_{A}$, назовем глобализатором решетки вложсений $\mathscr{I}$. Очевидно, что $g_{\mathscr{I}}$ есть вложение множества $\bigcup_{A \in H} A$ в множество $\bigcup_{B \in G} B$.

Пусть $\mathscr{A}$ - некоторая локально конечная алгебра и $\operatorname{Sub}_{\omega}(\mathscr{A})-$ совокупность всех основных множеств конечно порожденных подалгебр алгебры $\mathscr{A}$. Тогда $\operatorname{Sub} \omega(\mathscr{A})$ является решеткой подмножеств основного множества алгебры $\mathscr{A}$. Если $h-$ некоторое вложение алгебры $\mathscr{A}$ в какую-либо локально конечную алгебру $\mathscr{B}$, то $\mathscr{I}_{h}=\{h|C|$ $\left.C \in \operatorname{Sub}_{\omega}(\mathscr{A})\right\}$ является решеткой вложений решетки подмножеств $\operatorname{Sub}_{\omega}(\mathscr{A})$ в решетку подмножеств $\operatorname{Sub}_{\omega}(\mathscr{B})$. При этом глобализатор $g_{\mathscr{I}_{h}}$ решетки вложений $h$ совпадает с вложением $h$.

Под решеткой аләебр $\mathscr{H}$ будем понимать совокупность алгебр, основные множества которых образуют решетку подмножеств некоторого множества, если при этом включение $A \subseteq B$ между этими множествами влечет то, что алгебра с основным множеством $A$ является подалгеброй алгебры с основным множеством $B$. Для решетки алгебр $\mathscr{H}$ естественным образом определяется алгебра $\mathscr{H}$ с основным множеством $\bigcup\{A \mid A$ - основное множество некоторой алгебры из $\mathscr{H}\}$ так, что любая алгебра из $\mathscr{H}$ является подалгеброй алгебры $\mathscr{H}$. Пусть $\mathscr{H}$ и $\mathscr{G}$ - некоторые решетки алгебр и $\mathscr{I}$ - решетка вложений решетки алгебр $\mathscr{H}$ в решетку алгебр $\mathscr{G}$. Тогда очевидно, что глобализатор $g_{\mathscr{I}}$ будет вложением алгебры $\bigcup \mathscr{H}$ в алгебру $\mathscr{G}$.

В силу этих замечаний для любого локально конечного йонссоновского условного многообразия $\mathscr{K}$ если $U_{\mathscr{K}}\left(\aleph_{0}\right)$ - счетно-универсально-гомогенная $\mathscr{K}$-алгебра, то категория $\overleftrightarrow{\mathscr{K}}$ однозначно определяется решетками вложений решетки $\operatorname{Sub}_{\omega}\left(U_{\mathscr{K}}\left(\aleph_{0}\right)\right)$ в самою себя. То есть категория $\overleftrightarrow{\mathscr{K}}$ однозначно определяется парой $\left\langle\operatorname{Sub}_{\omega}\left(U_{\mathscr{K}}\left(\aleph_{0}\right)\right) ; \mathscr{E}(\mathscr{K})\right\rangle$, где $\mathscr{E}(\mathscr{K})$ - всевозможные изоморфизмы между алгебрами из $\operatorname{Sub}_{\omega}\left(U_{\mathscr{K}}\left(\aleph_{0}\right)\right)$.

ТеОРема 4.2 [42]. Пусть $\mathscr{K}_{1}, \mathscr{K}_{2}$ - локально конечные йонссоновские условные многообразия. Тогда $\mathscr{K}_{1}$ и $\mathscr{K}_{2}$ условно рационально эквивалентны в том и только том случае, когда существует биекиия основного множества алгебры $U_{\mathscr{K}_{1}}\left(\aleph_{0}\right)$ на основное множсество алгебры $U_{\mathscr{K}_{2}}\left(\aleph_{0}\right)$, сопрягающая парь $\left\langle\operatorname{Sub}_{\omega}\left(U_{\mathscr{K}_{1}}\left(\aleph_{0}\right)\right) ; \mathscr{E}\left(\mathscr{K}_{1}\right)\right\rangle$ u $\left\langle\operatorname{Sub}_{\omega}\left(U_{\mathscr{K}_{2}}\left(\aleph_{0}\right)\right) ; \mathscr{E}\left(\mathscr{K}_{2}\right)\right\rangle$, или, что то же самое, когда условно рачионально әквивалентны алгебры $U_{\mathscr{K}_{1}}\left(\aleph_{0}\right)$ и $U_{\mathscr{K}_{2}}\left(\aleph_{0}\right)$.

Таким образом, некоторая совокупность конечных подмножеств $\operatorname{Sub}_{\omega}\left(U_{\mathscr{K}}\left(\aleph_{0}\right)\right)$ некоторого счетного множества и некоторая совокупность $\mathscr{E}(\mathscr{K})$ биекций между этими 
подмножествами играют роль инвариантов для отношения условной рациональной эквивалентности между локально конечными йонссоновскими условными многообразиями. В связи с этим естественным становится вопрос описания совокупностей $H$ конечных подмножеств некоторого счетного множества $A$ и совокупностей $S$ биекций между этими подмножествами таких, что для некоторого локально конечного йонссоновского условного многообразия $\mathscr{K}$ на множестве $A$ определима алгебра $U U_{\mathscr{K}}\left(\aleph_{0}\right)$ такая, что $H=\operatorname{Sub}_{\omega}\left(U_{\mathscr{K}}\left(\aleph_{0}\right)\right)$ и $S=\mathscr{E}(\mathscr{K})$.

Наряду с условиями а)-ж) на пары $\langle H ; S\rangle$, рассмотренными в п. 1 настояшей главы, рассмотрим также следуюшие.

3) Для любого конечного подмножества $B$ множества $A$ сушествует $C \in H$ такое, что $B \subseteq C$ (наименьшее такое $C$ обозначим $H_{B}$ ), при этом сушествует функция $f: \omega \rightarrow \omega$ такая, что если $|B| \leqslant n$, то $\left|H_{B}\right| \leqslant f(n)$. Кроме того, для некоторого $a \in A$ множество $\{a\}$ входит в $H$.

и) Для любого $h \in S$ сушествует решетка $\mathscr{I}$ вложений решетки множеств $H$ самой в себя такая, что $h \in \mathscr{I}, \mathscr{I} \subseteq S, \bigcup_{\varphi \in \mathscr{I}} \operatorname{dom} \varphi=A$ и $\bigcup_{\varphi \in \mathscr{I}} \operatorname{rang} \varphi=A$.

к) Для любых $h_{1}, h_{2} \in S$ если $\operatorname{dom} h_{1}=\operatorname{dom} h_{2}$, то существуют $p_{1}, p_{2} \in S$ такие, что $\operatorname{rang} h_{1}=\operatorname{dom} p_{1}, \operatorname{rang} h_{2}=\operatorname{dom} p_{1}$ и $p_{1} h_{1}=p_{2} h_{2}$.

Очевидно, что для любого локально конечного йонссоновского условного многообразия пара $\left\langle\operatorname{Sub}_{\omega}\left(U_{\mathscr{K}}\left(\aleph_{0}\right)\right) ; \mathscr{E}(\mathscr{K})\right\rangle$ удовлетворяет условиям а)-к). Имеет место и обратное.

Теорема 4.3 [42]. Для решетки $Н$ подмножеств некоторого счетного множества $A$ и совокупности $S$ биекиий между множествами из $Н$ существует локально конечное йонссоновское условное многообразие $\mathscr{K}$ такое, что $H=$ $\operatorname{Sub}_{\omega}\left(U_{\mathscr{K}}\left(\aleph_{0}\right)\right)$ u $S=\mathscr{E}(\mathscr{K})$ тогда и только тогда, когда пара $\langle H ; S\rangle$ удовлетворяет условиям а) $-\mathrm{K})$.

Тем самым, теорема 4.3 полностью описывает системы инвариантов (в виде решеток конечных подмножеств и некоторых совокупностей биекций между этими подмножествами) для отношения условно рациональной эквивалентности между локально конечными йонссоновскими условными многообразиями.

Заключая эту тему, заметим, что в силу равенств $C T(\mathscr{A})=T(\mathscr{A})$ для дискриминаторных алгебр условно рациональная эквивалентность последних равносильна их рациональной эквивалентности. В работе [43] доказано, что это же самое имеет место для локально конечных дискриминаторных многообразий, и описаны инварианты (так называемые остовы многообразий) этих отношений для класса всех локально конечных дискриминаторных многообразий.

3. Инварианты отношения схожести между конечными алгебрами. Согласно следствию 3.3 конечные алгебры $\mathscr{A}_{1}$ и $\mathscr{A}_{2}$ схожи тогда и только тогда, когда инверсные полугрупшы Iso* $\mathscr{A}_{1}$ и Iso* $\mathscr{A}_{2}$ изоморфины (причем так, что идемпотенты полугрупшы Iso $\mathscr{A}_{1}$, соответствуюшие одноэлементным подалгебрам алгебры $\mathscr{A}_{1}$, переходят при этом изоморфизме в идемпотенты полугрупшы Iso $\mathscr{A}_{2}$, соответствующие одноэлементньм подалгебрам алгебры $\mathscr{A}_{2}$, и обратно).

Таким образом, для описания инвариантов отношения схожести на конечных универсальных алгебрах $\mathscr{A}$ необходимо абстрактное (с точностью до изоморфизма) описание класса полугрупп вида Iso* $\mathscr{A}$ для любых конечных алгебр $\mathscr{A}$.

Заметим, что инверсная полугруппа Iso $\mathscr{A}$ обладает нулем $(\varnothing)$ и единицей $(\mathrm{id} \mathscr{A})$. 
Пусть $\mathscr{B}$ некоторая инверсная полугруппа с нулем 0 и единицей 1 . Пусть $E=$ $\left\{a a^{-1} \mid a \in \mathscr{B}\right\}$ - совокупность ее идемпотентов. Здесь $a^{-1}-$ инверсньй к $a$ элемент из $\mathscr{B}$. Через $\mathscr{B}^{\prime}$ обозначим $\mathscr{B} \backslash\{0\}$.

Рассмотрим следующие условия 1)-3) на инверсную полугруппу $\mathscr{B}$.

1) Для любого $g \in \mathscr{B}^{\prime}$ сушествует идемпотент $e \in E$ такой, что для любого $b \in \mathscr{B}^{\prime}$ равенство $b g^{-1} g=b g^{-1}$ равносильно равенству $b g^{-1}=b e$.

Для любого $e \in E$ через $C(e)$ обозначим совокупность идемпотентов полугруппы $\mathscr{B}$, не превосходящих идемпотент $e$ (относительно традиционного порядка на $E$ : $\left.e_{1} \leqslant e_{2} \leftrightarrow e_{1} e_{2}=e_{1}\right)$ и компактных в решетке идемпотентов полугруппы $\mathscr{B}$.

2 ) Для любого $e \in E$ и любого отображения $F$ множества $C(e)$ в $\mathscr{B}$ такого, что:

а) для $h \in C(e)$ имеет место равенство $h=F(h) \cdot F(h)^{-1}$,

б) для $h_{1}, h_{2} \in C(e)$ имеет место равенство $h_{1} h_{2} F\left(h_{1}\right)=h_{2} h_{1} F\left(h_{2}\right)$,

найдется $f \in \mathscr{B}$ такой, что $f \cdot f^{-1}=e$ и для любого $h \in C(e)$ имеет место равенство $h F(h)=h f$.

Нетрудно заметить, что условие 1) соответствует переводу на язык инверсных полугрупп принципа неподвижных точек полугрупшы Iso $\mathscr{A}$, а условие 2) - принципа глобализации.

Идемпотент $е$ инверсной полугруппы $\mathscr{B}$ с нулем 0 назовем строго минимальным, если $e \neq 0, e$ минимален относительно порядка на ненулевых идемпотентах полугруппы $\mathscr{B}$ и если для любого $g \in \mathscr{B}$ из равенства $g g^{-1}=g^{-1} g=e$ вытекает равенство $g=e$.

Через $E_{S}$ обозначим совокупность всех строго минимальных идемпотентов полугрупшы $\mathscr{B}$.

Подмножество $U \subseteq E_{S}$ назовем замкнутылм, если для любых $e \in U$ и $g \in \mathscr{B}$ элемент $g^{-1} e g$ либо равен 0 , либо также лежит в $U$.

Фиксируем некоторое замкнутое подмножество $U$ строго минимальных идемпотентов полугрупшы $\mathscr{B}$ и рассмотрим следующее условие на пару $\langle\mathscr{B} ; U\rangle$.

3) Для любых $e_{1}, e_{2} \in U$ сушествует $g \in \mathscr{B}$ такой, что $g g^{-1}=e_{1}$ и $g^{-1} g=e_{2}$.

Условие 3) соответствует принципу одноэлементных подалгебр для полугруппы Iso $^{*} \mathscr{A}$.

Для любой универсальной алгебры $\mathscr{A}$ инверсная полугруппа Iso $\mathscr{A}$ обладает нулем и единицей, совокупность ее идемпотентов является алгебраической решеткой, и если $U$ - совокупность идемпотентов полугрупшы Iso $\mathscr{A}$, соответствуюших одноэлементным подалгебрам алгебры $\mathscr{A}$, то $U$ является замкнутым подмножеством множества строго минимальных идемпотентов полугруппы Iso $\mathscr{A}$, a пара $\langle$ Iso $\mathscr{A}, U\rangle$ удовлетворяет условиям 1)-3).

Верно и обратное. Варьируя доказательство теоремы 1.12, можно доказать следующее.

ТеОРема 4.4 [38]. Для любой инверсной полугруппь $\mathscr{B}$ с нулем и единицей, совокупность идемпотентов которой образует алгебраическую решетку, а также для некоторого замкнутого подмножсества $U$ совокупности ее строго минимальных идемпотентов, для которых выполнены условия 1)-3), существует универсальная алгебра $\mathscr{A}$ такая, что $\mathscr{B} \cong$ Iso $\mathscr{A}$ и элементам из $U$ соответствуют при этом изоморфизме в точности одноэлементные подалгебры алгебpbl $\mathscr{A}$.

Таким образом, имеет место 
СЛЕДСТВИЕ 4.2 [38]. Класс типов изоморфизма пар $\langle\mathscr{B}, U\rangle$, где $\mathscr{B}-$ конечная инверсная полугруппа с нулем и единичей, идемпотенты которой образуют решетку, $U$ - замкнутое подмножество строго минимальных идемпотентов полугруппь $\mathscr{B}$, а пара $\langle\mathscr{B}, U\rangle$ удовлетворяет условиям 1), 3), - играет роль инвариантов для отношения схожсести на конечньх универсальных алгебрах.

\section{Глава 5. \\ Классификация конечных алгебр по их вычислительным возможностям}

1. Шкала вычислительных потенциалов конечных алгебр. Как было замечено выше, совокупность $C T(\mathscr{A})$ условно термальных функций алгебры $\mathscr{A}$ есть совокупность функций, для которых существуют программы их вычислений, составленные из простейших программ (соответствующих сигнатурньм функциям алгебры $\mathscr{A}$ ) с помошњю оператора суперпозиции и условного оператора. В силу этого условно рационально эквивалентные алгебры $\mathscr{A}_{1}$ и $\mathscr{A}_{2}$ (т.е. алгебры, для которых совокупности этих функций $C T\left(\mathscr{A}_{1}\right)$ и $C T\left(\mathscr{A}_{2}\right)$ совпадают по модулю их сопряжения некоторой биекцией между основными множествами алгебр $\mathscr{A}_{1}$ и $\left.\mathscr{A}_{2}\right)$ естественно рассматривать как алгебры, обладающие одинаковым вьчислительным потенциалом. В главе 3 было доказано, что полугруппы Iso $\mathscr{A}$ внутренних изоморфизмов конечных алгебр являются инвариантами отношения условной рациональной эквивалентности этих алгебр, а в главе 4 приведено описание подполугрупп полугрупп частичных преобразований конечных множеств, имеюших вид Iso $\mathscr{A}$ для конечных алгебр. Эти результаты позволяют ставить вопрос о числе $n$-элементных алгебр $(n \in \omega)$, обладающих различным вычислительным потенциалом, пытаться каталогизировать такие алгебры, изучать сравнительную силу вычислительных потенциалов и т. п.

Будем далее считать, что рассматриваемые $n$-элементные алгебры заданы на основном множестве $\{0,1, \ldots, n-1\}$. На совокупности $C_{n}=\{C T(\mathscr{A}) \mid \mathscr{A}-n$-элементная алгебра $\}$ введем отношение эквивалентности $\sim: C T\left(\mathscr{A}_{1}\right) \sim C T\left(\mathscr{A}_{2}\right)$ тогда и только тогда, когда алгебры $\mathscr{A}_{1}$ и $\mathscr{A}_{2}$ условно рационально эквивалентны, т.е. сушествует перестановка $\pi$ множества $\{0,1, \ldots, n-1\}$, которая сопрягает совокупности функций $C T\left(\mathscr{A}_{1}\right)$ и $C T\left(\mathscr{A}_{2}\right)$. Через $C(n)$ обозначим фактор-множество $C_{n} / \sim$. Таким образом, мошность множества $C(n)$ соответствует числу попарно условно рационально не эквивалентных $n$-элементных алгебр, т.е. числу $n$-элементных алгебр, имеюших различньй вычислительный потенциал. Обозначим $|C(n)|$ как $S(n)$. Через $\leqslant$ обозначим отношение частичного порядка на $C(n)$, индуцированное отношением теоретико-множественного включения между элементами из $C_{n}$ при факторизации по отношению . Естественно следуюшее определение.

ОПРЕДЕЛЕНИЕ 5.1. Частично упорядоченное множество $\langle C(n) ; \leqslant\rangle$ назовем $ш к a-$ лой вычислительных потенциалов $n$-элементных алгебр.

Прежде всего заметим, что в отличие от классификации $n$-элементных алгебр, основанной на совпадении клонов их термальных функций (напомним, что в силу результата Е. Поста [58] число таких различных клонов на двухэлементном множестве счетно, а в силу результата Ю. И. Янова и А. А. Мучника [66] на $n$-элементном множестве, при $n \geqslant 3,-$ континуально), классификация $n$-элементных алгебр по их вьгислительным потенциалам конечна (число $S(n)$ различных вычислительных потенциалов та- 
ких алгебр не превышает числа попарно не сопряженных полугрупп внутренних изоморфизмов алгебр, заданных на множестве $\{0,1, \ldots, n-1\})$.

Следующий результат дает довольно грубую оценку числа $S(n)$.

ТЕОРема 5.1 [39]. Для любого натурального $n \geqslant 4$

$$
S(n) \leqslant 2^{n+\left(\frac{n}{e}\right)^{n-[\sqrt{n}]+1 / 2} \cdot e^{3([\sqrt{n}]+1 / 2)} .}
$$

Для малых $n$ числа $S(n)$ посчитаны в работе [39]: $S(2)=5, S(3)=53$. В приватном сообшении П. Джипсена указано, что им с помощью компютера получено равенство $S(4)=22610$. Заметим, что грубая оценка числа $S(4)$, вытекающая из теоремы 5.1 , имеет вид $S(4) \leqslant 2^{7770}$.

В работе [39] автора и в работе Б. Чаконя, Т. Говалковой [14] составлены каталоги представителей классов -эквивалентности двух- и трехэлементных алгебр, т.е. каталоги двух- и трехэлементных алгебр, обладаюших попарно различными вычислительньми потенциалами и такими, что вычислительньй потенщиал любой двух-, трехэлементной алгебры совпадает с вычислительным потенциалом одной из закаталогизированных алгебр. Для примера приведем подобный каталог для $n=2$ :

$$
\begin{gathered}
\mathscr{A}_{1}=\langle\{0,1\} ;+, \neg\rangle, \mathscr{A}_{2}=\langle\{0,1\} ; \neg\rangle, \mathscr{A}_{3}=\langle\{0,1\} ;+\rangle, \\
\mathscr{A}_{4}=\langle\{0,1\} ; \vee\rangle, \quad \mathscr{A}_{5}=\langle\{0,1\} ; \varnothing\rangle,
\end{gathered}
$$

здесь +- сложение $\bmod 2, \vee, \neg-$ стандартные булевы функции на $\{0,1\}$, а сигнатура алгебры $\mathscr{A}_{5}$ пуста.

В связи с грубостью оценки числа $S(n)$ в теореме 5.1 представляет интерес ее улучшение.

В отличие от совокупности клонов функций на $n$-элементном множестве, образуюшей решетку, шкала вычислительных потенщиалов $n$-элементных алгебр, вообще говоря, таковой не является.

ТеОРема 5.2 [48]. а) Для $n=2$ и 3 шкаль вычислительных потенииалов $n$-элементных алгебр являются решетками, для $n \geqslant 6$ они решетками не являются.

б) Для любой конечной решетки $L$ существуют натуральное $n$ и элементь $a, b \in C(n)$ такие, что интервал $[a, b]$ в шкале $\langle C(n) ; \leqslant\rangle$ является решеткой, в которую изоморфно вложима решетка $L$.

Шкалы $\langle C(2) ; \leqslant\rangle$ и $\langle C(3) ; \leqslant\rangle$, вычисленные в работе [48], приведены на рис. 1.

Представляет интерес дальнейшее изучение свойств шкал вычислительных потенциалов $n$-элементных алгебр.

2. Алгебры, условно рационально эквивалентные классическим алгебрам. Вполне естественными представляются вопросы описания на языке инвариантов $\langle$ Sub $\mathscr{A}$; Iso $\mathscr{A}\rangle$ конечных универсальных алгебр $\mathscr{A}$, условно рационально эквивалентных классическим: групшам, кольцам, полям, решеткам, полурешеткам, булевьм алгебрам и др., т.е. описание алгебр, имеюших вычислительньй потенциал, совпадающий с вычислительным потенциалом перечисленных классических алгебр. Подобные описания были получены автором в работах [39], [40], [47] для конечных алгебр, условно рашионально эквивалентных полурешеткам, решеткам (в частности, дистрибутивным и модулярным), булевым алгебрам, полям и унарам. В качестве примера 

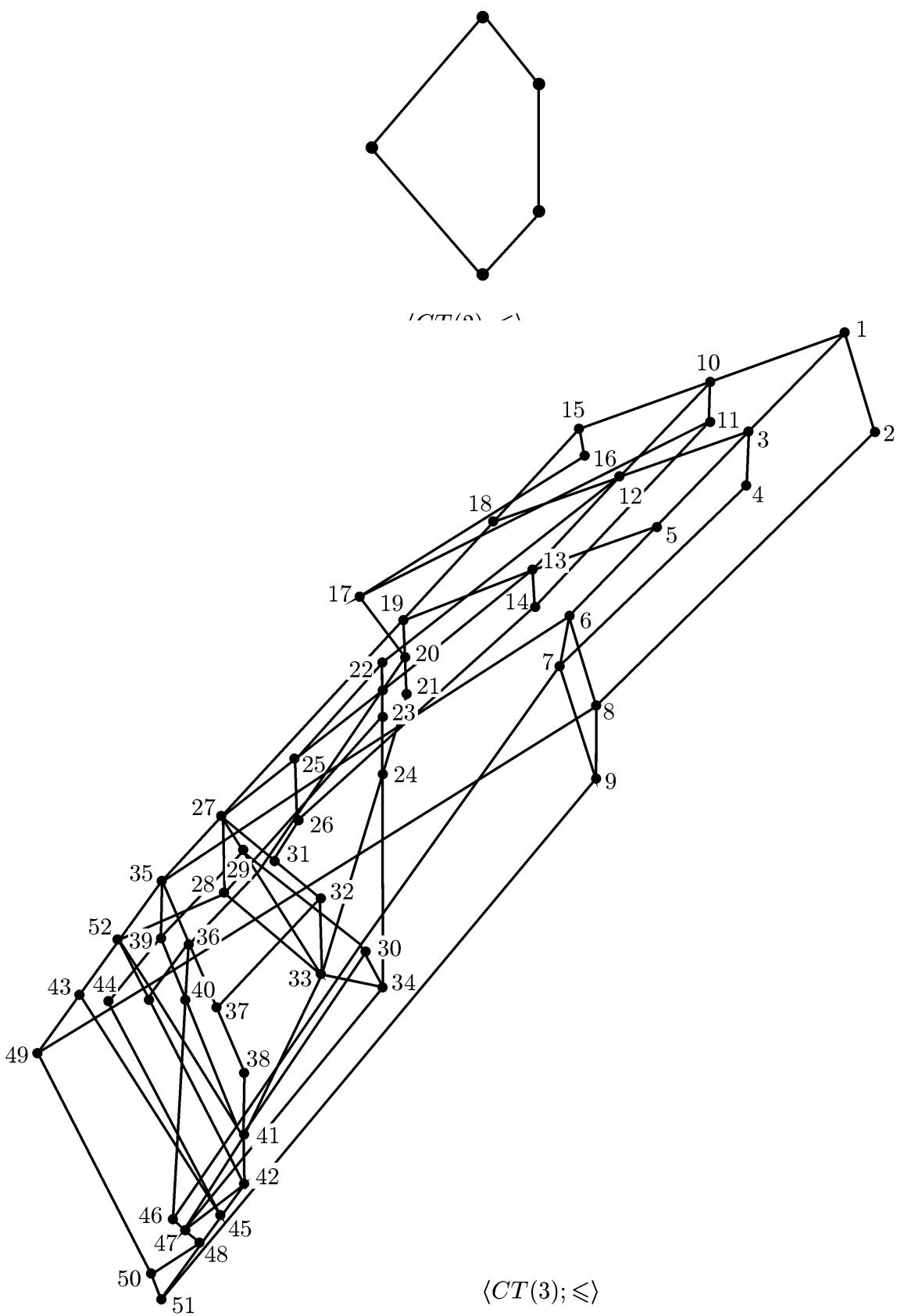

Рис. 1

приведем ниже описание инвариантов $\langle\operatorname{Sub} \mathscr{A} ;$ Iso $\mathscr{A}\rangle$ конечных алгебр $\mathscr{A}$, условно рационально эквивалентных полурешеткам и полям.

Напомним, что для любой алгебры $\mathscr{A}$ и любых ее элементов $a_{1}, \ldots, a_{n}$ через $\left\langle a_{1}, \ldots, a_{n}\right\rangle_{\mathscr{A}}$ обозначается подалгебра алгебры $\mathscr{A}$, порожденная элементами $a_{1}, \ldots, a_{n}$. Через $\operatorname{Sub}_{n} \mathscr{A}$ обозначим совокупность всех $n$-элементных подалгебр ал- 
гебры $\mathscr{A}$, через $P_{n}(A)$ - совокупность всех $n$-элементных подмножеств множества $A$, и пусть далее $A$ будет основньм множеством алгебры $\mathscr{A}$.

Рассмотрим следуюшие условия на пару $\langle\mathrm{Sub} \mathscr{A} ;$ Iso $\mathscr{A}\rangle$ для произвольной конечной алгебры $\mathscr{A}$.

$\left(\mathrm{sl}_{1}\right) \mathrm{Sub}_{1} \mathscr{A}=P_{1}(A)$, т.е. все однопорожденные подалгебры алгебры $\mathscr{A}$ одноэлементны.

$\left(\mathrm{sl}_{2}\right)$ Для любых $a, b \in \mathscr{A}$ если $a \neq b$, то $\langle a, b\rangle_{\mathscr{A}} \in \mathrm{Sub}_{2} \mathscr{A}$, либо $\langle a, b\rangle_{\mathscr{A}} \in \mathrm{Sub}_{3} \mathscr{A}$.

$\left(\mathrm{sl}_{3}\right)$ Для любых $\mathscr{B}_{1}, \mathscr{B}_{2} \in \mathrm{Sub}_{2} \mathscr{A}$ сушествует, причем единственный, изоморфизм $g \in$ Iso $\mathscr{A}$ такой, что rang $g=\mathscr{B}_{2}, \operatorname{dom} g=\mathscr{B}_{1}$.

$\left(\mathrm{sl}_{4}\right)$ Для любых $a, b, a^{\prime}, b^{\prime} \in \mathscr{A}$ если $\langle a, b\rangle_{\mathscr{A}},\left\langle a^{\prime}, b^{\prime}\right\rangle_{\mathscr{A}} \in \mathrm{Sub}_{3} \mathscr{A}$, то сушествуют ровно два изоморфизма $g_{1}, g_{2} \in$ Iso $\mathscr{A}$ такие, что dom $g_{1}=\operatorname{dom} g_{2}=\langle a, b\rangle_{\mathscr{A}}, \operatorname{rang} g_{1}=$ rang $g_{2}=\left\langle a^{\prime}, b^{\prime}\right\rangle_{\mathscr{A}}$. При этом если $\{a, b, c\}=\langle a, b\rangle_{\mathscr{A}},\left\{a^{\prime}, b^{\prime}, c^{\prime}\right\}=\left\langle a^{\prime}, b^{\prime}\right\rangle_{\mathscr{A}}$, то $g_{1}(c)=$ $g_{2}(c)$ и $\langle a, c\rangle_{\mathscr{A}},\langle b, c\rangle_{\mathscr{A}} \in \mathrm{Sub}_{2} \mathscr{A}$.

$\left(\mathrm{sl}_{5}\right)$ Сушествует не более одной (с точностью до изоморфизма) трехэлементной подалгебры алгебры $\mathscr{A}$, которая не является двухпорожденной, и если $\{a, b, c\} \in$ $\operatorname{Sub}_{3} \mathscr{A},\{a, b\},\{b, c\},\{a, c\} \in \mathrm{Sub}_{2} \mathscr{A}$ и $g \in \operatorname{Iso} \mathscr{A}$ таково, что $\operatorname{dom} g=\{a, b\}$, $\operatorname{rang} g=\{b, c\}, g(a)=b, g(b)=c$, то сушествует $h \in \operatorname{Iso} \mathscr{A}$ такой, что $\operatorname{dom} h=\{a, b\}$, rang $h=\{a, c\}, h(a)=a, h(c)=c . \quad$ Кроме того, для любых $a, b, c \in \mathscr{A}$ если $\{a, b\},\{b, c\} \in \operatorname{Sub}_{2} \mathscr{A}$ и сушествует $g \in \operatorname{Iso} \mathscr{A}$ такой, что $\operatorname{dom} g=\{a, b\}, \operatorname{rang} g=\{b, c\}$ и $g(a)=b, g(b)=c$, то $\{a, b, c\} \in \mathrm{Sub}_{3} \mathscr{A}$.

$\left(\mathrm{sl}_{6}\right)$ Для любых $a, b, c \in \mathscr{A}$ если $\langle a, b\rangle_{\mathscr{A}} \in \mathrm{Sub}_{3} \mathscr{A}$ и $\langle a, c\rangle_{\mathscr{A}},\langle b, c\rangle_{\mathscr{A}} \in \mathrm{Sub}_{2} \mathscr{A}$, $c \notin\langle a, b\rangle_{\mathscr{A}}$, то $\langle a, b, c\rangle \in \mathrm{Sub}_{4} \mathscr{A}$. Если при этом $\langle a, b\rangle_{\mathscr{A}}=\{a, b, d\}$, то сушествует $h \in \mathrm{Iso} \mathscr{A}$ такой, что $\operatorname{dom} h=\langle d, c\rangle_{\mathscr{A}}, \operatorname{rang} h=\langle a, c\rangle_{\mathscr{A}}$ и $h(d)=a, h(c)=c$.

Пусть далее $|\mathscr{A}|=m$.

$\left(\mathrm{sl}_{7}\right)$ Для любых $n \leqslant m, b_{1}, \ldots, b_{n}, c \in \mathscr{A}$ таких, что $c \in\left\langle b_{1}, \ldots, b_{n}\right\rangle_{\mathscr{A}}$, существует отображение $f$ множества $m=\{0,1, \ldots, m-1\}$ в совокупность $P(A)$ такое, что $f(0)=$ $\left\{b_{1}, \ldots, b_{n}\right\}$, для любого $0<r \leqslant m-1$ и любого $a^{\prime} \in f(r) \backslash f(r-1)$ найдутся $a^{\prime \prime}, a^{\prime \prime \prime} \in f(r-1)$ такие, что $a^{\prime} \in\left\langle a^{\prime \prime}, a^{\prime \prime \prime}\right\rangle_{\mathscr{A}}$, и при этом $c \in f(m-1)$.

$\left(\mathrm{sl}_{8}\right)$ Если биекция $h$ между множествами $B_{1}, B_{2} \in \mathrm{Sub} \mathscr{A}$ такова, что для любых $a, b \in B_{1} h \mid\langle a, b\rangle_{\mathscr{A}} \in$ Iso $\mathscr{A}$, то $h \in$ Iso $\mathscr{A}$.

Tеорема 5.3 [40]. вивалентна некоторой полурешетке (имеет вычислительныи потенциал, совпадающий с вьчислительньм потенциалом некоторой полурешетки) тогда и только тогда, когда пара $\langle\mathrm{Sub} \mathscr{A}$, Iso $\mathscr{A}\rangle$ удовлетворяет условиям $\left(\mathrm{sl}_{1}\right)-\left(\mathrm{sl}_{8}\right)$.

ТеОрема 5.4 [47]. Конечная универсальная алгебра А условно рационально эквивалентна некоторому полю тогда и только тогда, когда:

$\left(\mathrm{f}_{1}\right)|\mathscr{A}|=p^{n}$ для некоторых простого р и натурального $n$,

$\left(\mathrm{f}_{2}\right)$ любое отображсение из Isо $\mathscr{A}$ продолжимо до автоморфизма алгебры $\mathscr{A} ;$

$\left(\mathrm{f}_{3}\right)$ группа Aut $\mathscr{A}$ - ииклическая группа порядка $n$;

$\left(\mathrm{f}_{4}\right)$ подалгебрь алгебры $\mathscr{A}$ суть неподвижные точки подгрупп группь Aut $\mathscr{A} ;$

$\left(\mathrm{f}_{5}\right)$ если $H$ - подгруппа группь Aut $\mathscr{A}$, состоящая из әлементов порядка $\mathrm{m}$ $(m-$ делитель $n)$, то мощность множества неподвижных точек әлементов из $Н$ есть $p^{n / m}$. 
Представляет интерес описание в терминах $\langle\operatorname{Sub} \mathscr{A}$, Iso $\mathscr{A}\rangle$ конечных алгебр $\mathscr{A}$, вычислительный потенциал которых совпадает с вычислительным потенциалом некоторой группы, кольца.

В заключение отметим также следующий факт.

ТЕОРема 5.5 [40]. а) Отношение условной рациональной эквивалентности на полурешетках совпадает с отношением изоморфизма, т.е. неизоморфные полурешетки обладают различным вычислительным потенциалом.

б) Отношение условной рациональной эквивалентности на решетках совпадает с отношением рациональной әквивалентости, и при этом две решетки условно рационально эквивалентны тогда и только тогда, когда они изоморфнь либо двойственнь друг другу.

в) Отношение условной рациональной эквивалентности на булевьх алгебрах совпадает с отношением изоморфизма.

\section{Глава 6.}

\section{Обобщения условных термов}

В настоящей главе будут рассмотрены некоторые обобщения понятия условного терма на основе вариации понятия условия. Интерес к этим обобщениям основан, с одной стороны, на варьировании понятия программы вычисления на универсальной алгебре, а с другой - на вопросах описания функций, заданных на основном множестве универсальной алгебры и коммутирующих с различными полугруппами преобразований алгебры (см. теорему 3.2).

1. Элементарно условные термы. Далее в этом пункте под формулой будем понимать некоторую формулу исчисления предикатов первого порядка (фиксированной функциональной сигнатуры) - элементарную формулу. Полньлм набором элементарных условий сигнатуры $\sigma$ назовем любой набор $\left\{\Phi_{1}(\bar{x}), \ldots, \Phi_{k}(\bar{x})\right\}$ формул этой сигнатуры такой, что формула $\forall \bar{x} \bigvee_{i=1}^{k} \Phi_{i}(\bar{x})$ является тождественно истинной, а для любых различных $l, m \leqslant k$ формулы $\Phi_{l}(\bar{x}) \& \Phi_{m}(\bar{x})$ не выполнимы.

ОПРЕДЕЛЕНИЕ 6.1. Понятие элементарно условного терма сигнатуры $\sigma$ вводится с помошњю следуюшей индукции:

а) все переменные являются элементарно условными термами;

б) если $t_{1}(\bar{x}), \ldots, t_{k}(\bar{x})$ - элементарно условные термы сигнатуры $\sigma$ и $f\left(x_{1}, \ldots, x_{k}\right)$ $\in \sigma$, то $f\left(t_{1}(\bar{x}), \ldots, t_{k}(\bar{x})\right)$ - также элементарно условный терм сигнатуры $\sigma ;$

в) если $t_{1}(\bar{x}), \ldots, t_{m}(\bar{x})$ - элементарно условные термы и $\left\{\Phi_{1}(\bar{x}), \ldots, \Phi_{m}(\bar{x})\right\}$ - полный набор элементарных условий сигнатуры $\sigma$, то

$$
t(\bar{x})=\left\{\begin{array}{l}
\Phi_{1}(\bar{x}) \rightarrow t_{1}(\bar{x}) \\
\ldots \ldots \ldots \ldots \ldots \\
\Phi_{m}(\bar{x}) \rightarrow t_{m}(\bar{x})
\end{array}\right.
$$

также является элементарно условным термом этой сигнатуры;

г) любой элементарно условный терм строится за конечное число шагов согласно правилам а)-в). 
Если $\mathscr{A}$ - универсальная алгебра сигнатуры $\sigma$, то любой элементарно условньй терм $t(\bar{x})$ данной сигнатуры естественньм образом определяет на основном множестве алгебры $\mathscr{A}$ некоторую әлементарно условно термальную функиию. Укажем эту интерпретацию лишь для случая в) определения 6.1: если $\bar{b}, a \in \mathscr{A}$ и элементарно условньй терм $t(\bar{x})$ определен согласно правилу в), то $\mathscr{A} \models t(\bar{b})=a$ тогда и только тогда, когда для некоторого $l \leqslant m \mathscr{A} \models \Phi_{l}(\bar{b})$ и $\mathscr{A} \models t_{l}(\bar{b})=a$.

Совокупность всех элементарно условно термальных функций на алгебре $\mathscr{A}$ обозначим как $\operatorname{ECT}(\mathscr{A})$. Очевидно, что $C T(\mathscr{A}) \subseteq E C T(\mathscr{A})$.

$\mathrm{C}$ помошю аналога нормальной формы условных термов можно заметить, что элементарно условно термальные функции суть кусочно термальные, т.е. для любой такой функции $t\left(x_{1}, \ldots, x_{n}\right)$ на алгебре $\mathscr{A}$ существует разбиение $\mathscr{A}^{n}$ на множества $T_{1}, \ldots, T_{l}$ (для некоторого $l \in \omega$ ), определимые в $\mathscr{A}$ элементарными формулами, такое, что $t$, ограниченное на каждое из $T_{i}(i \leqslant l)$, совпадает с некоторой термальной на $\mathscr{A}$ функщией.

ОПРЕДЕлЕнИЕ 6.2. Равенство двух элементарно условных термов $t_{1}\left(\bar{x}_{1}\right)=t_{2}\left(\bar{x}_{2}\right)$ назовем элементарно условным тождеством, и истинность этого тождества на алгебре $\mathscr{A}$ будем интерпретировать как совпадение элементов $t_{1}\left(\bar{a}_{1}\right)$ и $t_{2}\left(\bar{a}_{2}\right)$ для любых наборов $\bar{a}_{1}$ и $\bar{a}_{2}$ элементов из $\mathscr{A}$, естественньм образом согласованных между собой. Класс всех универсальных алгебр фиксированной сигнатуры, на которых истинна некоторая совокупность элементарно условных тождеств, назовем әлементарно условным многообразием.

Нетрудно показать справедливость следующего утверждения.

ТЕОРема 6.1 [41]. Элементарно условные многообразия суть элементарные (аксиоматизируемые) классы алгебр, включающие в себя одноэлементную алгебру соответствующей сигнатуры.

Аналогично определению 1.2 рациональной эквивалентности и определению 3.1 условной рациональной эквивалентности с заменой классов функций $T(\mathscr{A})$ и соответственно $C T(\mathscr{A})$ на $E C T(\mathscr{A})$ дается определение элементарно условной рациональной эквивалентности как классов, так и отдельных алгебр.

Для класса $\mathscr{K}$ универсальных алгебр сигнатуры $\sigma$ через $\mathscr{K}^{l}$ обозначим класс обогащений $\mathscr{K}$-алгебр за счет добавления в сигнатуру новых функциональных символов $q_{t}(\bar{x})$ для каждого элементарно условного терма $t(\bar{x})$ сигнатуры $\sigma$, интерпретируя эти символы $q_{t}$ на $\mathscr{K}^{l}$-алгебрах с помощью элементарно условно термальных функций $t$. Через $\sigma^{l}$ обозначим сигнатуру класса $\mathscr{K}^{l}$, а через $\mathscr{A}^{l}-$ соответствующее обогащение алгебры $\mathscr{A}$. Класс $\mathscr{K}^{l}$ выделяется среди класса всех алгебр сигнатуры $\sigma^{l}$ аксиомами класса $\mathscr{K}$ и элементарно условными тождествами вида $q_{t}(\bar{x})=t(\bar{x})$. Если $\varphi-$ некоторое вложение $\mathscr{K}$-алгебры $\mathscr{A}$ в $\mathscr{K}$-алгебру $\mathscr{A}_{1}$, то $\varphi$ остается вложением алгебры $\mathscr{A}^{l}$ в

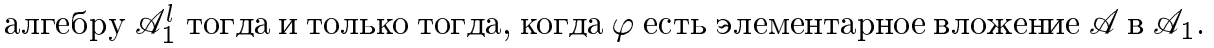

Пусть $\mathscr{G}_{1}$ и $\mathscr{G}_{2}$ - две категории универсальных алгебр и $\mathscr{G}_{1}^{\prime}$ и $\mathscr{G}_{2}^{\prime}-$ некоторые полные подкатегории категорий $\mathscr{G}_{1}$ и $\mathscr{G}_{2}$ соответственно. Скажем, что пары категорий универсальных алгебр $\left\langle\mathscr{G}_{1}, \mathscr{G}_{1}^{\prime}\right\rangle$ и $\left\langle\mathscr{G}_{2}, \mathscr{G}_{2}^{\prime}\right\rangle$ әквивалентны, если существует изоморфизм $G$ категории $\mathscr{G}_{1}$ на категорию $\mathscr{G}_{2}$, коммутирующий со стирающими функторами этих категорий и такой, что $G\left(\mathscr{G}_{1}^{\prime}\right)=\mathscr{G}_{2}^{\prime}$. 
Через $\stackrel{\vec{K}}{\longrightarrow}$ для любого класса алгебр $\mathscr{K}$ обозначим категорию, объектами которой являются $\mathscr{K}$-алгебры, а морфизмами - элементарные вложения $\mathscr{K}$-алгебр друг в друга плюс эпиморфизмы $\mathscr{K}$-алгебр на одноэлементную алгебру, если последняя входит в $\mathscr{K}$ (категорию әлементарных вложсений класса $\mathscr{K}$ ). В силу замеченного выше категории $\stackrel{l_{\mathscr{K}}}{\longrightarrow} \stackrel{\mathscr{K}^{l}}{\longrightarrow}$ эквивалентны.

ОПРЕДЕЛЕНИЕ 6.3. Два элементарных условных многообразия $\mathscr{K}_{1}$ и $\mathscr{K}_{2}$ назовем әквивалентными относительно элементарных влоэсений внутри классов их элементарно условно термальных подаләебр, если эквивалентны пары категорий

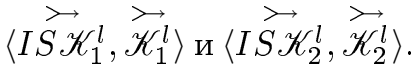

Следующее утверждение является параллелью теореме 3.1 об условно ращионально эквивалентных условных многообразиях.

Теорема 6.2 [41]. Два элементарно условных многообразия $\mathscr{K}_{1}$ и $\mathscr{K}_{2}$ элементарно условно рационально әквивалентны тогда и только тогда, когда они әквивалентны относительно әлементарных вложсений внутри классов их элементарно условно термальных подалгебр.

Существуют примеры, показьваюшие, что эквивалентности категорий элементарных вложений элементарно условных многообразий для их элементарно условно рациональной эквивалентности недостаточно.

Класс элементарно условно термальных функций допускает естественную характеризацию как класс функций, коммутируюших с некоторыми полугруппами преобразований алгебр (подобно теореме 3.2 для условно термальных функций).

Теорема 6.3 [50]. Для любой конечной алгебры $А$ функиия $f$ на основном множестве алгебры $\mathscr{A}$ является әлементарно условно термальной тогда и только тогда, когда подалгебры алгебры $\mathscr{A}$ замкнуты относительно $f$ и $f$ коммутирует со всеми автоморфизмами алгебры $\mathscr{A}$.

Утверждение теоремы не может быть обобщено на случай равномерно локально конечных алгебр даже конечной сигнатуры.

СЛЕДСТВИЕ 6.1 [50]. Для любьх двух конечных алгебр $\mathscr{A}_{1}$ и $\mathscr{A}_{2}$ с общим основным множсеством следующие условия әквивалентны:

1) $\operatorname{ECT}\left(\mathscr{A}_{1}\right)=\operatorname{ECT}\left(\mathscr{A}_{2}\right)$;

2) Sub $\mathscr{A}_{1}=\operatorname{Sub} \mathscr{A}_{2}$, Aut $\mathscr{A}_{1}=$ Aut $\mathscr{A}_{2}$.

В силу того, что для любой алгебры $\mathscr{A}$ клон $C T(\mathscr{A})$ условно термальных функций на $\mathscr{A}$ является конечно порожденньм расширением клона $T(\mathscr{A})$ ее термальных функций $\left(C T(\mathscr{A})=T\left(\mathscr{A}^{d}\right)\right)$, естественен подобньй вопрос о клоне $E C T(\mathscr{A})$ элементарно условно термальных функций алгебры $\mathscr{A}$.

В работе [55] построена бесконечная алгебра $\mathscr{A}$ такая, что клон $E C T(\mathscr{A})$ не является конечно порожденным расширением клона $T(\mathscr{A})$. В работе [50] доказано, что для любой конечной алгебры $\mathscr{A}$ клон $\operatorname{ECT}(\mathscr{A})$ является конечно порожденным расширением клона $T(\mathscr{A})$. 


\section{2. Позитивно условные и $\exists^{+}$-условные термы.}

ОПРЕДЕЛЕНИЕ 6.4. а) Позитивным условием сигнатуры $\sigma$ назовем любую конечную систему равенств между термами сигнатуры $\sigma$

$$
P(\bar{x})=\left\{\begin{array}{c}
t_{1}^{1}(\bar{x})=t_{2}^{1}(\bar{x}) \\
\ldots \ldots \ldots \cdots \\
t_{1}^{n}(\bar{x})=t_{2}^{n}(\bar{x})
\end{array} .\right.
$$

б) Понятие позитивно условного терма для алгебры $\mathscr{A}$ определяется индукцией, аналогичной определению условного и элементарно условного терма с заменой в соответствуюших определениях правила в) на следуюшее:

в) если $\left\{P_{1}(\bar{x}), \ldots, P_{k}(\bar{x})\right\}$ - совокупность позитивных условий, $t_{1}(\bar{x}), \ldots, t_{k}(\bar{x})-$ позитивно условные термы для алгебры $\mathscr{A}$ и при этом $\mathscr{A} \models \forall \bar{x}\left(\bigvee_{i=1}^{k} P_{i}(\bar{x})\right)$ и для любых $p \neq q \leqslant k$

$$
\mathscr{A} \models \forall \bar{x}\left(P_{p}(\bar{x}) \& P_{q}(\bar{x}) \rightarrow t_{p}(\bar{x})=t_{q}(\bar{x})\right),
$$

TO

$$
t(\bar{x})=\left\{\begin{array}{l}
P_{1}(\bar{x}) \rightarrow t_{1}(\bar{x}) \\
\ldots \ldots \ldots \ldots \\
P_{k}(\bar{x}) \rightarrow t_{k}(\bar{x})
\end{array}\right.
$$

также является позитивно условным термом для алгебры $\mathscr{A}$.

Любой позитивно условный терм для алгебры $\mathscr{A}$ естественным образом определяет на ее основном множестве позитивно условно термальную функцию. Совокупность всех позитивно условно термальных функций алгебры $\mathscr{A}$ обозначим $\operatorname{PCT}(\mathscr{A})$. Очевидны включения $T(\mathscr{A}) \subseteq P C T(\mathscr{A}) \subseteq C T(\mathscr{A})$ для любой алгебры $\mathscr{A}$.

Под внутренним гомоморфизмом алгебры $\mathscr{A}$ будем понимать гомоморфизм любой ее подалгебры на произвольную подалгебру алгебры $\mathscr{A}$. Полугруппу всех внутренних гомоморфизмов алгебры $\mathscr{A}$ обозначим как $\operatorname{Ihm} \mathscr{A}$.

Имеет место следуюшее утверждение.

ТЕОРема 6.4 [51]. Для любой конечной или равномерно локально конечной конечной сигнатуры алгебры $\mathscr{A}$ функиия $f$, заданная на основном множестве алгебры $\mathscr{A}$, является позитивно условно термальной тогда и только тогда, когда все подалгебры алгебры $\mathscr{A}$ замкнуты относительно $f$ и $f$ коммутирует со всеми внутренними гомоморфизмами алгебры $\mathscr{A}$.

Заметим, что все ограничения на алгебру $\mathscr{A}$ в формулировке теоремы сушественны.

СлЕДСТВИЕ 6.2 [51]. Для любых двух конечных либо равномерно локально конечных конечной сигнатуры алгебр $\mathscr{A}_{1}$ и $\mathscr{A}_{2}$ с общим основным множсеством следуюшие условия әквивалентны:

1) $\operatorname{PCT}\left(\mathscr{A}_{1}\right)=\operatorname{PCT}\left(\mathscr{A}_{2}\right)$;

2) $\operatorname{Sub} \mathscr{A}_{1}=\operatorname{Sub} \mathscr{A}_{2}, \operatorname{Ihm} \mathscr{A}_{1}=\operatorname{Ihm} \mathscr{A}_{2}$.

В работе [55] показано сушествование бесконечных алгебр $\mathscr{A}$ таких, что клон $\operatorname{PCT}(\mathscr{A})$ не является конечно порожденным расширением клона $T(\mathscr{A})$. Вопрос о том, будет ли для любой конечной алгебры $\mathscr{A}$ клон $P C T(\mathscr{A})$ являться конечно порожденным расширением клона $T(\mathscr{A})$, остается открытым. 
Под $\exists^{+}$- условием сигнатуры $\sigma$ будем понимать любую $\exists$-позитивную формулу $\Phi(\bar{x})$ исчисления предикатов первого порядка данной сигнатуры. Понятие $\exists^{+}$-yсловного терма определяется полностью аналогично определению позитивно условного терма с заменой в правиле в) позитивных условий на $\exists^{+}$-условия. Совокупность всех $\exists^{+}$-условных функиий на алгебре $\mathscr{A}$ обозначим как $\exists^{+} C T(\mathscr{A})$. Очевидны включения $\operatorname{PCT}(\mathscr{A}) \subseteq \exists^{+} C T(\mathscr{A}) \subseteq E C T(\mathscr{A})$. В то же время совокупности $C T(\mathscr{A})$ и $\exists^{+} C T(\mathscr{A})$ в общем случае не входят одна в другую.

Через End $\mathscr{A}$ обозначим полугруппу всех эндоморфизмов алгебры $\mathscr{A}$ в себя.

Имеет место следуюшее утверждение.

ТеОрема 6.5 [50]. Для любой конечной алгебры $\mathscr{A}$ и любой функиии $f$, заданной на основном множсестве алгебры $\mathscr{A}$, функиия $f$ является $\exists^{+}$-условно термальной на $\mathscr{A}$ тогда и только тогда, когда все подалгебры алгебрь $\mathscr{A}$ замкнутьи относительно $f$ u $f$ коммутирует со всеми әндоморфизмами алгебры $\mathscr{A}$.

Ограничение на конечность алгебры $\mathscr{A}$ в формулировке теоремы существенно.

СлЕДСТВИЕ 6.3 [50]. Для любих двух конечных алгебр $\mathscr{A}_{1}$ и $\mathscr{A}_{2}$ с общим основньмм множеством следуюшие условия әквивалентны:

1) $\exists^{+} C T(\mathscr{A})=\exists^{+} C T\left(\mathscr{A}_{2}\right)$;

2) $\operatorname{Sub} \mathscr{A}_{1}=\operatorname{Sub} \mathscr{A}_{2}$, End $\mathscr{A}_{1}=$ End $\mathscr{A}_{2}$.

В работе [50] показано существование бесконечных алгебр $\mathscr{A}$ таких, что клон $\exists^{+} C T(\mathscr{A})$ не является конечно порожденньм расширением клона $T(\mathscr{A})$. Вопрос о том, будет ли для любой конечной алгебры $\mathscr{A}$ клон $\exists^{+} C T(\mathscr{A})$ являться конечно порожденньм расширением клона $T(\mathscr{A})$, остается открытым.

Ряд иных фактов, связанных с условными термами, можно найти в работах [37], [46], [48], [49], [54], [55].

\section{СПИСОК ЛИТЕРАТУРЫ}

[1] В.А. Артамонов. Универсальные алгебры // Общая алгебра / ред. Л.А. Скорняков. T. 2. М.: Наука, 1990. C. 295-364.

[2] C. Bergman. Categorical equivalence of algebras with a majority term // Algebra Universalis. 1998. V. 40. № 2. P. 149-175.

[3] C. Bergman, J. Berman. Morita equivalence of almost-primal clones // J. Pure Appl. Algebra. 1996. V. 108. № 2. P. 175-201.

[4] E. W. Beth. On Padoa's method in the theory of definition // Konink. Nederl. Akad. Wetensch. Proceedings. Ser. A. 1953. V. 56. № 3. P. 330-339.

[5] Г. Биркгоф, Т. М. Барти. Современная прикладная алгебра. М.: Мир, 1976.

[6] Д. А. Бредихин. Инверсные полугруппы локальных автоморфизмов универсальных алгебр // Сиб. матем. журн. 1976. Т. 17. № 3. С. 490-507.

[7] S. Bulman-Fleming, H. Werner. Equational compactness in quasi-primal varieties // Algebra Universalis. 1977. V. 7. № 1. P. 33-46.

[8] S. Burris, R. McKenzie. Decidability and Boolean Representations. Providence, RI: Amer. Math. Soc., 1981. (Mem. Amer. Math. Soc. V. 246.)

[9] S. Burris, H. P. Sankappanavar. A Course in Universal Algebra. Berlin: Springer-Verlag, 1981.

[10] C. C. Chang, H. J. Keisler. Model Theory. Amsterdam / New York: North-Holland / Elsevier, 1973.

[11] D. M. Clark, P. H. Krauss. Boolean representation of congruence-distributive varieties // Preprint № 19/79. Kassel: Gesam-thochenschule, 1979.

[12] А. Клиффорд, Г. Престон. Алгебраическая теория полугрупп. М.: Мир, 1972. 
[13] П. Кон. Универсальная алгебра. М.: Мир, 1968.

[14] B. Csakany, T. Gavalcova. Three-element quasiprimal algebras // Studia Sci. Math. Hungar. 1981. V. 16. № 1-2. P. 237-238.

[15] А. Г. Курош. Лекции по общей алгебре. М.: Наука, 1973.

[16] A. L. Foster, A. F. Pixley. Semi-categorical algebras. I; II // Math. Z. 1964. V. 83. P. $147-169$; V. 85 . P. $169-184$.

[17] G. Gratzer. Universal Algebra. Berlin: Springer-Verlag, 1979.

[18] T. K. Hu. On the topological duality for primal algebra theory // Algebra Universalis. 1974. V. 1. № 1. P. 152-154.

[19] B. Jonsson. Homogeneous universal relational systems // Math. Scand. 1960. V. 8. № 1. P. $137-142$.

[20] B. Jonsson. Algebras whose congruence lattices are distributive // Math. Scand. 1967. V. 21. № 1. P. 110-121.

[21] B. Jonsson. Topics in Universal Algebra. Berlin: Springer-Verlag, 1972.

[22] B. Jonsson. Congruence distributive varieties // Math. Jap. 1995. V. 48. № 2. P. 353-401.

[23] S. MacLane. Categories for the Working Mathematician. Berlin: Springer-Verlag, 1971.

[24] А. И. Мальцев. Структурная характеристика некоторых классов алгебр // Докл. АН CCCP. 1958. T. 120. № 1. C. 29-32.

[25] А. И. Мальцев. Алгебраические системы. М.: Наука, 1970.

[26] R. N. McKenzie. On spectra and the negative solution of the decision problem for identities having a finite nontrivial model // J. Symbolic Logic. 1975. V. 40. P. 187-196.

[27] R. McKenzie. An algebraic version of categorical equivalence for varieties and more general algebraic categories // Logic and Algebra / ed. A. Ursini et al. New York: Marcel Dekker, 1996. P. 211-243. (Lecture Notes in Pure and Appl. Math. V. 180.)

[28] R. N. McKenzie, G. McNulty, W. Taylor. Algebras, Lattices, Varieties. V. 1. Montrey, CA: Wadsworth \& Brooks / Cole, 1987.

[29] G. Michler, R. Wille. Die primitiven Klassen arithmetischer Ringe // Math. Z. 1970. V. 113. P. 369-172.

[30] M. Morley, R. Vaught. Homogeneous universal models // Math. Scand. 1962. V. 11. № 1. P. 37-57.

[31] В. Л. Мурский. Существование конечного базиса тождеств и другие свойства "почти всех" конечных алгебр // Проблемы кибернетики. 1975. Т. 30. С. 43-56.

[32] А. Г. Пинус. Конгруэнц-модулярные многообразия алгебр. Иркутск: Изд-во ИрГУ, 1986.

[33] А. Г. Пинус. Основы универсальной алгебры. Новосибирск: Изд-во НГТУ, 1998.

[34] А. Г. Пинус. Булевы конструкции в универсальной алгебре // УМН. 1992. Т. 47. № 4. C. $145-180$.

[35] А. Г. Пинус. Об условных термах и тождествах на универсальных алгебрах // Вычислительные системы. 1996. № 156. С. 59-78.

[36] А. Г. Пинус. Характеризация условно термальных функций // Сиб. матем. журн. 1997. T. 38. № 1. С. 161-165.

[37] А. Г. Пинус. Условные термы и программы для вычислений на алгебрах // Вестник НГТУ. 1996. № 2. С. 145-151.

[38] А. Г. Пинус. Исчисление условных тождеств и условно рациональная эквивалентность // Алгебра и логика. 1998. Т. 37. № 4. С. 432-459.

[39] А. Г. Пинус. Об условно рационально эквивалентных алгебрах // Вычислителшные системы. 1999. № 165 . С. 3-30.

[40] А. Г. Пинус. Об алгебрах, условно рационально эквивалентных полурешеткам и булевым алгебрам // Сиб. матем. журн. 1998. Т. 39. № 1. С. 121-128.

[41] А. Г. Пинус. $N$-элементная вложимость и $n$-условные термы // Изв. вузов. Матем. 1999. № 1. C. 36-40.

[42] А. Г. Пинус. Йонссоновские локально конечные условные многообразия и условно рациональная эквивалентность // Сиб. матем. журн. 1998. Т. 39. № 4. С. 942-948.

[43] А. Г. Пинус. Об условно рационально эквивалентных дискриминаторных многообразиях // Изв. вузов. Матем. 1999. № 8. С. 54-59.

[44] А. Г. Пинус. Условная топология и определимые функции на универсалњных алгебрах // Сиб. матем. журн. 1999. Т. 40. №6. С. 1305-1312. 
[45] А. Г. Пинус. Об определимости конечных алгебр производными структурами // Изв. вузов. Матем. (в печати).

[46] А. Г. Пинус. Об алгебрах условно термальных функций // Алгебра и теория моделей. Новосибирск: Изд-во НГТУ, 1997. С. 123-130.

[47] А. Г. Пинус. Внутренние изоморфизмы и условно рациональная эквивалентность унарам и полям // Алгебра и теория моделей. Новосибирск: Изд-во НГТУ, 1997. С. 131-142.

[48] А. Г. Пинус, С.В.ЖКурков. О шкалах потенциалов вычислимости универсалшных алгебр // Труды Международной конференции "Галуа соответствия" (в печати).

[49] А. Г. Пинус. Об уравновешенных условных тождествах и условно полных условных многообразиях // Фунд. прикл. матем. (в печати).

[50] А. Г. Пинус. О функциях коммутирующих с полугруппами преобразований алгебр // Сиб. матем. журн. 2000. Т. 41. №6. С. 1409-1418.

[51] А. Г. Пинус. Внутренние гомоморфизмы и позитивно условные термы // Алгебра и логика. 2001. Т. 40. № 2. С. 158-173.

[52] A. G. Pinus. Boolean Consructions in Universal Algebras. Dordrecht: Kluwer, 1993.

[53] A. G. Pinus. Conditional terms and Skolem functions // Contributions to General Algebra. Klagenfurt: Johannes Heyn Verlag, 1999. P. 155-160.

[54] A. G. Pinus. Conditional terms and their applications // Algebra: Proceedings of the International Algebraic Conference of the Occasion of the 90th Birthday of A. G. Kurosh. Berlin: Walter de Gruyter, 2000. P. 291-299.

[55] A. G. Pinus. The positive and generalized discriminators don't exist // Discuss. Math. Gen. Algebra Appl. 2000. V. 20. № 1. P. 121-128.

[56] A. F. Pixley. Distributivity and permutability of congruence relations in equational classes of algebras // Proc. Amer. Math. Soc. 1963. V. 14. P. 105-109.

[57] A. F. Pixley. The ternary discriminator function in universal algebra // Math. Ann. 1971. V. 191. P. $167-180$.

[58] E. L. Post. The Two-Valued Iterative Systems of Mathematical Logic. Princeton: Princeton Univ. Press, 1941. (Ann. Math. Stud. V. 5.)

[59] G. B. Preston. Representations of inverse semigroups // J. London Math. Soc. 1954. V. 29. P. 411-419.

[60] R.W. Quackenbush. Structure theory for equational classes generated by quasi-primal algebras // Trans. Amer. Math. Soc. 1974. V. 187. №1. P. 127-145.

[61] Л.А. Скорняков. Элементы общей алгебры. М.: Наука, 1983.

[62] Д. М. Смирнов. Многообразия алгебр. Новосибирск: Наука, 1992.

[63] M. G. Stone. Subalgebra and automorphism structure in universal algebra // Preprint, 1973.

[64] В. В. Вагнер. Обобщенные группы // Докл. АН СССР. 1952. Т. 84. С. 1119-1122.

[65] H. Werner. Discriminator Algebras. Berlin: Academie-Verlag, 1978.

[66] Ю. И. Янов, А. А. Мучник. О существовании $k$-значных замкнутых классов, не имеющих конечного базиса // Докл. АН СССР. 1959. Т. 127. № 1. С. 44-46.

Новосибирский государственный

Поступила в редакцию технический университет

30.10 .2000 